\title{
Human-in-the-Loop Systems with Inner and Outer Feedback Control Loops: Adaptation, Stability Conditions, and Performance Constraints ${ }^{\star}$
}

\author{
Ehsan Arabi* and Tansel Yucelen ${ }^{\dagger}$ \\ University of South Florida \\ Rifat Sipahi ${ }^{\ddagger}$ \\ Northeastern University \\ Yildiray Yildiz ${ }^{\S}$ \\ Bilkent University
}

In this paper, we focus on human-in-the-loop physical systems with inner and outer feedback control loops. Specifically, our problem formulation considers that inner loop control laws use a model reference adaptive control approach to suppress the effect of system uncertainties such that the overall physical system operates close to its ideal behavior as desired in the presence of adverse conditions due to failures and/or modeling inaccuracies. Moreover, we consider that the outer loop control laws exist owing to employing either sequential loop closure and/or high-level guidance methods. As it is true in practice, in addition, humans are considered to inject commands directly to the outer loop dynamics in response to the changes in the physical system, where the outer loop commands affect inner loop dynamics in response to the commands received from the humans as well as in response to the changes in the physical system.

The presence of humans can result in system instability, even when the resulting physical system augmented with inner and outer feedback control loops yield to stable trajectories in the absence of humans. This paper addresses this problem by proving a sufficient stability condition for the overall physical system with human dynamics modeled as a linear timeinvariant system with human reaction time-delay, where this condition does not depend on system uncertainties similar to our recent theoretical results. Furthermore, inner loop system errors during the transient phase of adaptively suppressing system uncertainties can severely affect the human-outer loop interactions. We also address this issue by utilizing a recently proposed set-theoretic model reference adaptive control approach at the inner loop for enforcing a user-defined performance constraint on the norm of the system error trajectories, where we show how the selection of this constraint affects the overall physical system. Finally, the efficacy of our results is demonstrated through an illustrative numerical example for an adaptive flight control application with a Neal-Smith pilot model.

${ }^{*}$ E. Arabi is a Graduate Research Assistant of the Department of Mechanical Engineering and a Member of the Laboratory for Autonomy, Control, Information, and Systems (LACIS, http://lacis.eng.usf.edu/) at the University of South Florida, Tampa, FL 33620, USA (email: ehsanarabi@mail.usf.edu).

$\dagger \mathrm{T}$. Yucelen is an Assistant Professor of the Department of Mechanical Engineering and the Director of the Laboratory for Autonomy, Control, Information, and Systems (LACIS, http://lacis.eng.usf.edu/) at the University of South Florida, Tampa, FL 33620, USA (email: yucelen@usf.edu). In addition, he is an Adjunct Professor of the Department of Mechanical and Aerospace Engineering at the Missouri University of Science and Technology, Rolla, MO 65409, USA. T. Yucelen is also a Senior Member of the American Institute of Aeronautics and Astronautics and a Member of the National Academy of Inventors.

${ }^{\ddagger} \mathrm{R}$. Sipahi is a Professor at the Mechanical and Industrial Engineering Department and the Director of the Complex Dynamic Systems and Control Laboratory at Northeastern University, Boston, MA 02115, USA (email: rifat@coe.neu.edu).

$\S$ Y. Yildiz is an Assistant Professor of the Mechanical Engineering Department and the Director of the Systems Laboratory at the Bilkent University, Ankara, Turkey (email: yyildiz@bilkent.edu.tr).

${ }^{\star}$ This research was supported by the National Aeronautics and Space Administration under grant NNX15AM51A. 


\section{Introduction}

This paper focuses on human-in-the-loop physical systems with inner and outer feedback control loops. Building on our recent results documented in [1], our problem formulation considers that inner loop control laws use a model reference adaptive control approach to suppress the effect of system uncertainties such that the overall physical system operates close to its ideal behavior in the presence of adverse conditions due to failures and/or modeling inaccuracies (we refer to [1] and references therein for relevant literature). Specifically, the model reference adaptive control approach of this paper has the capability to enforce performance constraints on the transient system performance (see below), unlike the results in [1]. Moreover, it theoretically augments a general nominal dynamic compensator structure; that is, the nominal control law utilized in [1] becomes a special case of the nominal dynamic compensator considered in this paper. Here, we also explicitly consider outer loop control laws within our problem formulation. From an application standpoint, these outer loop control laws can exist owing to employing either sequential loop closure and/or high-level guidance methods. While the results in [1] can consider entire system dynamics to enforce objectives achieved by such methods, it is well-known that sequential loop closure approaches can ease the control design task through several low-order control laws (see, for example, $[2,3]$ and references therein) and/or one would like to simply add a guidance algorithm to an existing inner loop feedback control architecture.

As it is true in practice, here we also consider humans inject commands directly to the outer loop dynamics in response to the changes in the physical system, and in return, the outer loop commands affect inner loop dynamics in response to the commands received from the humans as well as in response to the changes in the physical system. In particular, the presence of humans can result in system instability especially due to human reaction time-delays (see, for example, $[1,4-8]$ and references therein), even when the resulting physical system augmented with inner and outer feedback control loops yield to stable trajectories in the absence of humans. In this paper, we address this problem by proving a sufficient stability condition for the overall physical system with human dynamics modeled as a linear time-invariant system with human reaction time-delay, where this condition does not depend on system uncertainties similar to the results in [1]. Furthermore, inner loop system errors during the transient phase of adaptively suppressing system uncertainties can severely affect the human-outer loop interactions. This paper also addresses this issue by utilizing a recently developed model reference adaptive control approach discussed next.

Specifically, with conventional model reference adaptive control algorithms like the one adopted in [1], only a conservative bound on the system errors can be theoretically developed; this bound depends on the bound on the system uncertainties. Thus, without a complete knowledge of the upper bound on the system uncertainties, the adaptively controlled overall system may exhibit unsatisfactory performance (i.e., large system error signal) resulting in poor human-physical system interaction. While a high adaptation gain can be used at all times as a remedy, such a choice is not always practically desirable. To overcome this limitation of conventional model reference adaptive control laws, the authors recently proposed the set-theoretic model reference adaptive control architecture in [9] for achieving time-invariant user-defined performance bounds. In $[10,11]$ this framework was further extended to guarantee time-varying user-defined performance bounds. The generalizations of the set-theoretic model reference adaptive control architecture to the unstructured system uncertainties, actuator failures, actuator dynamics were then studied in [12-15]. Within the scope of this paper, we use this new architecture in [9] for enforcing a user-defined performance constraint on the norm of the system error trajectories, where we explicitly show how the selection of this constraint affects the overall physical system. Finally, the efficacy of the overall human-in-the-loop physical system architecture of this paper with inner and outer feedback control loops is demonstrated through an illustrative numerical example for an adaptive flight control application with a Neal-Smith pilot model. 
The organization of this paper is as follows. Section II presents the problem formulation, where Section III discusses the stability and performance of the overall human-in-the-loop physical system architecture. Section IV presents the aforementioned illustrative numerical example, where the conclusions are finally drawn in Section V. We refer to appendices for the notation used in this paper as well as necessary definitions.

\section{Problem Formulation}

In this paper, we consider a human-machine system in which the machine behavior $y(t)$ is observed by the human. Based on this observation, the human then generates a decision and commands the actuator for tracking purposes. To improve the overall tracking performance with the human, instead of direct interaction of the human with the machine, we consider outer and inner control loops with which human commands are properly delegated to the machine to stabilize the error dynamics. To start the control design, consider the block diagram representation of the human-in-the-loop physical systems with inner and outer feedback control loops as given in Figure 1. Note that in this setting, the human input (i.e., the reference command) is what the human aims to achieve in a given task and the uncertain dynamical system is the physical system on which this task is being performed. The outer loop architecture then uses the initial command constructed by the human loop and generates the command signal that is fed into the inner loop. The inner loop architecture includes the uncertain dynamical system as well as the model reference adaptive controller components (i.e., the reference model, the parameter adjustment mechanism, and the controller).

In what follows, we provide detailed discussion for each of these three loops. Specifically, we consider the uncertain dynamics representing a physical system given by

$$
\dot{x}^{*}(t)=\left[\begin{array}{cc}
A_{\mathrm{p}} & 0_{n_{\mathrm{p}} \times n_{\phi_{\mathrm{p}}}} \\
G_{\mathrm{p}} & F_{\mathrm{p}}
\end{array}\right] x^{*}(t)+\left[\begin{array}{c}
B_{\mathrm{p}} \\
0_{n_{\mathrm{c}} \times m}^{\mathrm{T}}
\end{array}\right]\left(\Lambda u(t)+\delta_{\mathrm{p}}\left(x_{\mathrm{p}}(t)\right)\right),
$$

where $A_{\mathrm{p}} \in \mathbb{R}^{n_{\mathrm{p}} \times n_{\mathrm{p}}}, B_{\mathrm{p}} \in \mathbb{R}^{n_{\mathrm{p}} \times m}, F_{\mathrm{p}} \in \mathbb{R}^{n_{\phi_{\mathrm{p}}} \times n_{\phi_{\mathrm{p}}}}$, and $G_{\mathrm{p}} \in \mathbb{R}^{n_{\phi_{\mathrm{p}}} \times n_{\mathrm{p}}}$ are the system matrices, $u(t) \in \mathbb{R}^{m}$

is the control input, $\delta_{\mathrm{p}}: \mathbb{R}^{n_{\mathrm{p}}} \rightarrow \mathbb{R}^{m}$ is a system uncertainty, $\Lambda \in \mathbb{R}_{+}^{m \times m} \cap \mathbb{D}^{m \times m}$ is an unknown control effectiveness matrix, and we assume that the overall system is controllable. Letting $x^{*}(t)=\left[x_{\mathrm{p}}^{\mathrm{T}}(t), \phi_{\mathrm{p}}^{\mathrm{T}}(t)\right]^{\mathrm{T}} \in$ $\mathbb{R}^{n_{\mathrm{p}}+n_{\phi_{\mathrm{p}}}}$, where $x_{\mathrm{p}}(t) \in \mathbb{R}^{n_{\mathrm{p}}}$ is the primary measurable state vector and $\phi_{\mathrm{p}} \in \mathbb{R}^{n_{\phi_{\mathrm{p}}}}$ is the secondary measurable state vector, one can equivalently write (1) as

$$
\begin{aligned}
& \dot{x}_{\mathrm{p}}(t)=A_{\mathrm{p}} x_{\mathrm{p}}(t)+B_{\mathrm{p}} \Lambda u(t)+B_{\mathrm{p}} \delta_{\mathrm{p}}\left(x_{\mathrm{p}}(t)\right), \quad x_{\mathrm{p}}(0)=x_{\mathrm{p} 0}, \\
& \dot{\phi}_{\mathrm{p}}(t)=F_{\mathrm{p}} \phi_{\mathrm{p}}(t)+G_{\mathrm{p}} x_{\mathrm{p}}(t), \quad \phi_{\mathrm{p}}(0)=\phi_{\mathrm{p} 0} .
\end{aligned}
$$

Specifically, we design the controller at the inner loop based on the structure given by (2). We then utilize (3) to design an outer loop dynamic compensator for generating the command signal that is fed to the inner loop.

\section{A Inner Loop Architecture}

At the inner loop architecture, we consider the uncertain dynamical system given in (2) and assume that the system uncertainty $\delta_{\mathrm{p}}\left(x_{\mathrm{p}}(t)\right)$ is parameterized as

$$
\delta_{\mathrm{p}}\left(x_{\mathrm{p}}(t)\right)=W_{\mathrm{p}}^{\mathrm{T}}(t) \sigma_{\mathrm{p}}\left(x_{\mathrm{p}}(t)\right),
$$




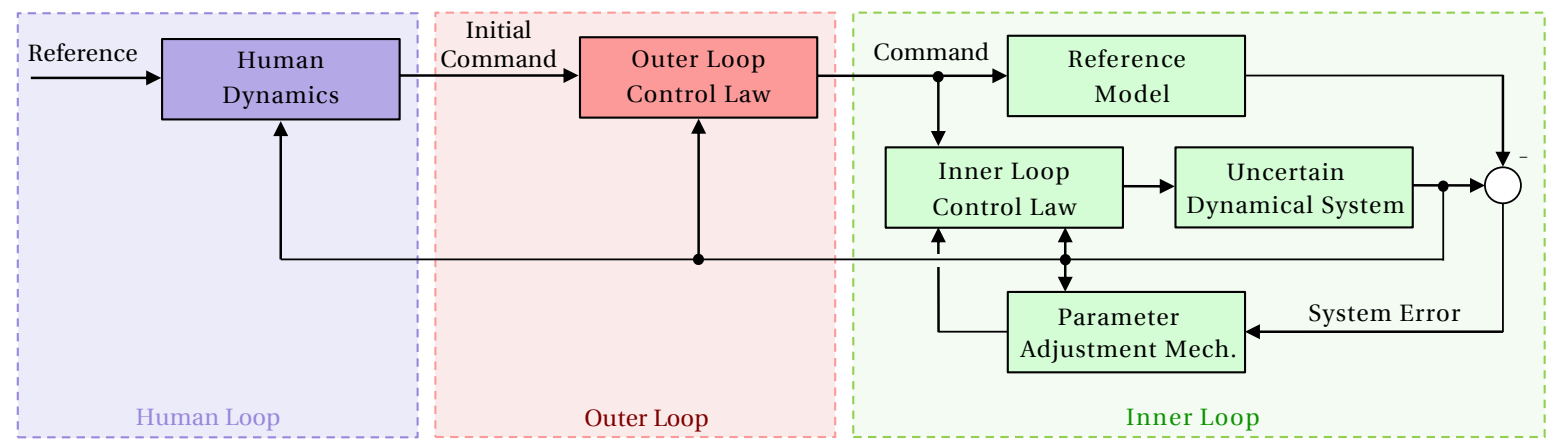

Figure 1. Block diagram of the human-in-the-loop model reference adaptive control architecture.

where $W_{\mathrm{p}}(t) \in \mathbb{R}^{s \times m}$ is a bounded unknown weight matrix (i.e., $\left\|W_{0}(t)\right\|_{\mathrm{F}} \leq w_{0}$ ) with a bounded time rate of change (i.e., $\left.\left\|\dot{W}_{0}(t)\right\|_{\mathrm{F}} \leq \dot{w}_{0}\right)$ and $\sigma_{\mathrm{p}}: \mathbb{R}^{n_{\mathrm{p}}} \rightarrow \mathbb{R}^{s}$ is a known basis function of the form $\sigma_{\mathrm{p}}\left(x_{\mathrm{p}}(t)\right)=$ $\left[\sigma_{\mathrm{p} 1}\left(x_{\mathrm{p}}(t)\right), \sigma_{\mathrm{p} 2}\left(x_{\mathrm{p}}(t)\right), \ldots, \sigma_{\mathrm{p} s}\left(x_{\mathrm{p}}(t)\right)\right]^{\mathrm{T}}$.

To address command following at the inner loop architecture, let $x_{\mathrm{c}}(t) \in \mathbb{R}^{n_{\mathrm{c}}}$ be the dynamic compensator state satisfying

$$
\begin{aligned}
\dot{x}_{\mathrm{c}}(t) & =A_{\mathrm{c}} x_{\mathrm{c}}(t)+B_{\mathrm{c}} e_{\mathrm{p}}(t), \quad x_{\mathrm{c}}(0)=x_{\mathrm{c} 0}, \\
z_{\mathrm{c}}(t) & =C_{\mathrm{c}} x_{\mathrm{c}}(t)+D_{\mathrm{c}} e_{\mathrm{p}}(t),
\end{aligned}
$$

where $A_{\mathrm{c}} \in \mathbb{R}^{n_{\mathrm{c}} \times n_{\mathrm{p}}}, B_{\mathrm{c}} \in \mathbb{R}^{n_{\mathrm{c}} \times n_{y}}, C_{\mathrm{c}} \in \mathbb{R}^{n_{\mathrm{z}} \times n_{\mathrm{c}}}, D_{\mathrm{c}} \in \mathbb{R}^{n_{z} \times n_{y}}, z(t) \in \mathbb{R}^{z}$ is the output of the dynamic compensator, $e_{\mathrm{p}}(t) \triangleq y(t)-c(t)$, and $y(t) \triangleq C_{\mathrm{p}} x_{\mathrm{p}}(t)$ with $C_{\mathrm{p}} \in \mathbb{R}^{n_{y} \times n_{\mathrm{p}}}$. We now consider the inner loop control law given by

$$
u(t)=u_{\mathrm{n}}(t)+u_{\mathrm{a}}(t)
$$

where $u_{\mathrm{n}}(t) \in \mathbb{R}^{m}$ and $u_{\mathrm{a}}(t) \in \mathbb{R}^{m}$ are the nominal and adaptive control laws, respectively. Furthermore, let the nominal control law be

$$
u_{\mathrm{n}}(t)=-K_{\mathrm{p}} x_{\mathrm{p}}(t)-K_{\mathrm{c}} z_{\mathrm{c}}(t)
$$

with $K_{\mathrm{p}} \in \mathbb{R}^{m \times n_{\mathrm{p}}}$ and $K_{\mathrm{c}} \in \mathbb{R}^{m \times n_{z}}$. Now, (2) can be augmented with (5) as

$$
\dot{x}(t)=A_{\mathrm{r}} x(t)+B_{\mathrm{r}} c(t)+B \Lambda\left(u_{\mathrm{a}}(t)+W^{\mathrm{T}}(t) \sigma(x(t), c(t))\right), \quad x(0)=x_{00},
$$

where $x(t) \triangleq\left[x_{\mathrm{p}}^{\mathrm{T}}(t), x_{\mathrm{c}}^{\mathrm{T}}(t)\right]^{\mathrm{T}} \in \mathbb{R}^{n}, n=n_{\mathrm{p}}+n_{\mathrm{c}}$, is the augmented state vector, $W(t) \triangleq\left[W_{\mathrm{p}}^{\mathrm{T}}(t),\left(\Lambda^{-1}\right.\right.$ $\left.\left.-I_{m \times m}\right)\left(K_{\mathrm{p}}+K_{\mathrm{c}} D_{\mathrm{c}} C_{\mathrm{p}}\right),\left(\Lambda^{-1}-I_{m \times m}\right) K_{\mathrm{c}} C_{\mathrm{c}},-\left(\Lambda^{-1}-I_{m \times m}\right) K_{\mathrm{c}} D_{\mathrm{c}}\right]^{\mathrm{T}} \in \mathbb{R}^{\left(s+n+n_{y}\right) \times m}$ is an unknown (aggregated) weight matrix, $\sigma(x(t), c(t)) \triangleq\left[\sigma_{\mathrm{p}}^{\mathrm{T}}\left(x_{\mathrm{p}}(t)\right), x_{\mathrm{p}}^{\mathrm{T}}(t), x_{\mathrm{c}}^{\mathrm{T}}(t), c(t)\right]^{\mathrm{T}} \in \mathbb{R}^{s+n+n_{y}}$ is a known (aggregated) basis function, $x_{00} \triangleq\left[x_{\mathrm{p} 0}^{\mathrm{T}}, x_{\mathrm{c} 0}^{\mathrm{T}}\right]^{\mathrm{T}}$,

$$
\begin{aligned}
A_{\mathrm{r}} \triangleq\left[\begin{array}{cc}
A_{\mathrm{p}}-B_{\mathrm{p}} K_{\mathrm{p}}-B_{\mathrm{p}} K_{\mathrm{c}} D_{\mathrm{c}} C_{\mathrm{p}} & -B_{\mathrm{p}} K_{\mathrm{c}} C_{\mathrm{c}} \\
B_{\mathrm{c}} C_{\mathrm{p}} & A_{\mathrm{c}}
\end{array}\right] \in \mathbb{R}^{n \times n}, \\
B_{\mathrm{r}} \triangleq\left[\begin{array}{c}
B_{\mathrm{p}} K_{\mathrm{c}} D_{\mathrm{c}} \\
-B_{\mathrm{c}}
\end{array}\right] \in \mathbb{R}^{n \times n_{y}},
\end{aligned}
$$




$$
B \triangleq\left[\begin{array}{ll}
B_{\mathrm{p}}^{\mathrm{T}} & 0_{n_{\mathrm{c}} \times m}^{\mathrm{T}}
\end{array}\right]^{\mathrm{T}} \in \mathbb{R}^{n \times m}
$$

Considering (9), let the adaptive control law be in the form given by

$$
u_{\mathrm{a}}(t)=-\hat{W}^{\mathrm{T}}(t) \sigma(x(t), c(t))
$$

where $\hat{W}(t) \in \mathbb{R}^{\left(s+n+n_{y}\right) \times m}$ is the estimate of $W(t)$. Following the set-theoretic model reference adaptive control architecture presented in [9] (see also [10-15]), let the update law for (13) be given by

$$
\dot{\hat{W}}(t)=\gamma \operatorname{Proj}_{\mathrm{m}}\left(\hat{W}(t), \phi_{d}\left(\|e(t)\|_{P}\right) \sigma(x(t)) e^{\mathrm{T}}(t) P B\right), \quad \hat{W}(0)=\hat{W}_{0}
$$

with $\hat{W}_{\max }$ being the projection norm bound. In (14), $\gamma \in \mathbb{R}_{+}$is the learning rate (i.e., adaptation gain), $P \in \mathbb{R}_{+}^{n \times n}$ is a solution of the Lyapunov equation given by

$$
0=A_{\mathrm{r}}^{\mathrm{T}} P+P A_{\mathrm{r}}+R
$$

with $R \in \mathbb{R}_{+}^{n \times n}$, and $e(t) \triangleq x(t)-x_{\mathrm{r}}(t)$ is the system error with $x_{\mathrm{r}}(t) \in \mathbb{R}^{n}$ being the reference state vector of a reference model dynamics at the inner loop that captures a desired inner loop dynamical system performance given by

$$
\dot{x}_{\mathrm{r}}(t)=A_{\mathrm{r}} x_{\mathrm{r}}(t)+B_{\mathrm{r}} c(t), \quad x_{\mathrm{r}}(0)=x_{\mathrm{r} 0} .
$$

Using (13), (14), and (16), the inner loop system error dynamics is given by

$$
\begin{aligned}
\dot{e}(t) & =A_{\mathrm{r}} e(t)-B \Lambda \tilde{W}^{\mathrm{T}}(t) \sigma(x(t), c(t)), \quad e(0)=e_{0}, \\
\dot{\tilde{W}}(t) & =\gamma \operatorname{Proj}_{\mathrm{m}}\left(\hat{W}(t), \phi_{d}\left(\|e(t)\|_{P}\right) \sigma(x(t), c(t)) e^{\mathrm{T}}(t) P B\right)-\dot{W}(t), \quad \tilde{W}(0)=\tilde{W}_{0},
\end{aligned}
$$

where $\tilde{W}(t) \triangleq \hat{W}(t)-W(t) \in \mathbb{R}^{\left(s+n+n_{y}\right) \times m}$ is the weight estimation error and $e_{0} \triangleq x_{00}-x_{\mathrm{r} 0}$. Once again, we note that the unknown weight matrix $W(t)$ and its derivative have unknown upper bounds (i.e., $\|W(t)\|_{\mathrm{F}} \leq w$ and $\|\dot{W}(t)\|_{\mathrm{F}} \leq \dot{w}$ with unknown $w$ and $\left.\dot{w}\right)$.

Comment 1 (Set-theoretic Model Reference Adaptive Control). The update law given by (14) for the set-theoretic model reference adaptive control architecture can be derived by considering the following energy function

$$
V(e, \tilde{W})=\phi\left(\|e\|_{P}\right)+\gamma^{-1} \operatorname{tr}\left[\left(\tilde{W} \Lambda^{1 / 2}\right)^{\mathrm{T}}\left(\tilde{W} \Lambda^{1 / 2}\right)\right]
$$

As shown in [9], the time derivative of this energy function is upper bounded by

$$
\dot{V}(e(t), \tilde{W}(t)) \leq-\frac{1}{2} \alpha_{1} V(e, \tilde{W})+\alpha_{2}
$$

where $\alpha_{1} \triangleq \frac{\lambda_{\min }(R)}{\lambda_{\max }(P)}, d \triangleq 2 \gamma^{-1} \tilde{w} \dot{w}\|\Lambda\|_{2}, \alpha_{2} \triangleq \frac{1}{2} \alpha_{1} \gamma^{-1} \tilde{w}^{2}\|\Lambda\|_{2}+d$, and $\tilde{w}=\hat{W}_{\max }+w$. In particular, (20) is sufficient to conclude that $V(e, \tilde{W})$ is upper bounded. Hence, one can now conclude with $\left\|e_{0}\right\|_{P}<\epsilon$ that the pair $(e(t), \tilde{W}(t))$ is bounded and the system error satisfies the strict bound given by

$$
\|e(t)\|_{P}<\epsilon, \quad t \geq 0
$$




\section{B Outer Loop Architecture}

We now construct the outer loop control law for (3). Specifically, consider the dynamic compensator given by

$$
\begin{aligned}
\dot{\phi}_{\mathrm{c}}(t) & =F_{\mathrm{c}} \phi_{\mathrm{c}}(t)+G_{\mathrm{c}} \eta_{\mathrm{p}}(t), \quad \phi_{\mathrm{p}}(0)=\phi_{\mathrm{p} 0}, \\
c(t) & =H_{\mathrm{c}} \phi_{\mathrm{c}}(t)-J_{\mathrm{c}} \eta_{\mathrm{p}}(t), \\
\eta_{\mathrm{p}}(t) & =M_{\mathrm{p}} \phi_{\mathrm{p}}(t)-c_{0}(t),
\end{aligned}
$$

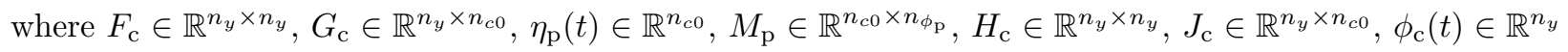
is the outer loop state vector, $c_{0}(t) \in \mathbb{R}^{n_{c 0}}$ is the initial command signal produced by the human, which is the input to the outer loop architecture, and $c(t) \in \mathbb{R}^{n_{y}}$ is the generated command at the outer loop as shown in Figure 1. As discussed earlier, these outer loop dynamics can exist owing to employing either sequential loop closure and/or high-level guidance methods.

Now by letting $\phi(t)=\left[x_{\mathrm{r}}^{\mathrm{T}}(t), \phi_{\mathrm{p}}^{\mathrm{T}}(t), \phi_{\mathrm{c}}^{\mathrm{T}}(t)\right]^{\mathrm{T}} \in \mathbb{R}^{n_{\phi}}, n_{\phi}=n+n_{\phi_{\mathrm{p}}}+n_{y}$, one can write (3), (16), and (22) in a compact form as

$$
\dot{\phi}(t)=F_{\mathrm{r}} \phi(t)+G_{\mathrm{r}} c_{0}(t)+G e(t), \quad \phi(0)=\phi_{0},
$$

where

$$
\begin{aligned}
F_{\mathrm{r}} \triangleq & {\left[\begin{array}{ccc}
A_{\mathrm{r}} & -B_{\mathrm{r}} J_{\mathrm{c}} M_{\mathrm{p}} & B_{\mathrm{r}} H_{\mathrm{c}} \\
G_{\mathrm{p}} N & F_{\mathrm{p}} & 0 \\
0 & G_{\mathrm{c}} M_{\mathrm{p}} & F_{\mathrm{c}}
\end{array}\right] \in \mathbb{R}^{\left(n+n_{\phi_{\mathrm{p}}}+n_{y}\right) \times\left(n+n_{\phi_{\mathrm{p}}}+n_{y}\right)}, } \\
G_{\mathrm{r}} & \triangleq\left[\begin{array}{c}
B_{\mathrm{r}} J_{\mathrm{c}} \\
0 \\
-G_{\mathrm{c}}
\end{array}\right] \in \mathbb{R}^{\left(n+n_{\phi_{\mathrm{p}}}+n_{y}\right) \times n_{c 0}}, \quad G \triangleq\left[\begin{array}{c}
0 \\
G_{\mathrm{p}} N \\
0
\end{array}\right] \in \mathbb{R}^{\left(n+n_{\phi_{\mathrm{p}}}+n_{y}\right) \times n},
\end{aligned}
$$

with $N=\left[I_{n_{\mathrm{p}} \times n_{\mathrm{p}}}, 0_{n_{\mathrm{p}} \times n_{\mathrm{c}}}\right]$. Note that $F_{\mathrm{r}}$ should be made a Hurwitz matrix by design to capture stability when $c_{0}(t)$ is bounded a-priori in the absence of uncertainties; this is discussed in the next comment.

Comment 2 (Bounded Solution of $(25)$ ). If $c_{0}(t)$ is bounded, then it follows from $F_{\mathrm{r}}$ being Hurwitz and $e(t)$ being bounded (see Comment 1 ) that the solution $\phi(t)$ to $(25)$ is bounded. Yet, since humans make decisions in response to the system states received from the dynamical system as shown in the human loop part of Figure $1, c_{0}(t)$ cannot be assumed to be a-priori bounded signal. We refer to the next subsection and Section III for more details concerning this point.

\section{Human Loop Architecture}

For the human loop, we consider a general class of linear human models with constant time-delay [1]

$$
\begin{aligned}
\dot{\xi}(t) & =A_{\mathrm{h}} \xi(t)+B_{\mathrm{h}} \theta(t-\tau), \quad \xi(0)=\xi_{0} \\
c_{0}(t) & =C_{\mathrm{h}} \xi(t)+D_{\mathrm{h}} \theta(t-\tau),
\end{aligned}
$$

where $\xi(t) \in \mathbb{R}^{n_{\xi}}$ is the internal human state vector, $\tau \in \mathbb{R}_{+}$is the human reaction time-delay, $A_{\mathrm{h}} \in \mathbb{R}^{n_{\xi} \times n_{\xi}}$, $B_{\mathrm{h}} \in \mathbb{R}^{n_{\xi} \times n_{\mathrm{r}}}, C_{\mathrm{h}} \in \mathbb{R}^{n_{c 0} \times n_{\xi}}$, and $D_{\mathrm{h}} \in \mathbb{R}^{n_{c 0} \times n_{\mathrm{r}}}$. Here, the input to the human dynamics is given by 


$$
\theta(t)=r(t)-E_{\mathrm{h}} \phi_{\mathrm{p}}(t)
$$

where $\theta(t) \in \mathbb{R}^{n_{\mathrm{r}}}$, and $r(t) \in \mathbb{R}^{n_{\mathrm{r}}}$ is the bounded reference signal. In (30), $E_{\mathrm{h}} \in \mathbb{R}^{n_{\mathrm{r}} \times n}$ selects the appropriate states to be compared with $r(t)$. Note that the dynamics given by (28), (29) and (30) captures a wide range of linear time-invariant human models with time-delay including Neal-Smith model and its extensions [18-22] and is also utilized as-is by the authors of [1] in their analysis.

\section{Stability and Performance}

Based on the structure of the inner, outer, and human loops presented in the previous section, we now analyze the closed-loop system performance and show how the system error at the inner loop affects the human loop. We then demonstrate the effectiveness of the set-theoretic model reference adaptive control architecture at the inner loop for guaranteeing a user-defined performance constraint on the norm of system error trajectory without any knowledge of the upper bound on the system uncertainties. This ultimately results in an acceptable human performance in accomplishing a given task. For this purpose, letting $x_{0}(t) \triangleq$ $\left[\phi^{\mathrm{T}}(t), \xi^{\mathrm{T}}(t)\right]^{\mathrm{T}} \in \mathbb{R}^{n_{0}}, n_{0} \triangleq n_{\phi}+n_{\xi}$, one can write the dynamics in (25) and (28) as

$$
\dot{x}_{0}(t)=A_{0} x_{0}(t)+A_{1} x_{0}(t-\tau)+B_{0} r(t-\tau)+B_{1} e(t), \quad x_{0}(t)=\psi_{0}(t) \quad \text { for } \quad t \in[-\tau, 0],
$$

where $\psi_{0}(t) \in \mathbb{R}^{n_{0}}$ is the initial condition and

$$
\begin{aligned}
& A_{0} \triangleq\left[\begin{array}{cc}
F_{\mathrm{r}} & G_{\mathrm{r}} C_{\mathrm{h}} \\
0 & A_{\mathrm{h}}
\end{array}\right] \in \mathbb{R}^{n_{0} \times n_{0}}, \quad A_{1} \triangleq\left[\begin{array}{cc}
-G_{\mathrm{r}} D_{\mathrm{h}} E_{\mathrm{h}} N_{0} & 0 \\
-B_{\mathrm{h}} E_{\mathrm{h}} N_{0} & 0
\end{array}\right] \in \mathbb{R}^{n_{0} \times n_{0}}, \\
& B_{0} \triangleq\left[\begin{array}{c}
G_{\mathrm{r}} D_{\mathrm{h}} \\
B_{\mathrm{h}}
\end{array}\right] \in \mathbb{R}^{n_{0} \times n_{\mathrm{r}}} \quad, \quad B_{1} \triangleq\left[\begin{array}{c}
G \\
0
\end{array}\right] \in \mathbb{R}^{n_{0} \times n},
\end{aligned}
$$

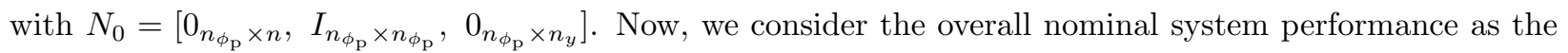
case where there is no uncertainty in the system at the inner loop. In other words, the overall performance of the human interacting with the physical system in the absence of any uncertainties is viewed as the ideal behavior represented by

$$
\dot{\hat{x}}_{0}(t)=A_{0} \hat{x}_{0}(t)+A_{1} \hat{x}_{0}(t-\tau)+B_{0} r(t-\tau), \quad \hat{x}_{0}(t)=\hat{\psi}_{0}(t) \quad \text { for } \quad t \in[-\tau, 0] .
$$

Now letting $\tilde{x}(t) \triangleq x_{0}(t)-\hat{x}_{0}(t)$ and using (31) and (34), the error dynamics can be written as

$$
\dot{\tilde{x}}(t)=A_{0} \tilde{x}(t)+A_{1} \tilde{x}(t-\tau)+B_{1} e(t), \quad \tilde{x}(t)=\psi(t) \quad \text { for } \quad t \in[-\tau, 0]
$$

where $\psi(t) \triangleq \psi_{0}(t)-\hat{\psi}_{0}(t)$.

Comment 3 (Stability of Nominal System $(e(t)=0)$ ). Setting $e(t)=0$ in $(35)$, the nominal system is obtained as

$$
\dot{\tilde{x}}(t)=A_{0} \tilde{x}(t)+A_{1} \tilde{x}(t-\tau) .
$$

Notice here that the delay term appears only in the state but not in the derivative of the state. This class of dynamics are known as retarded type [23-25], which exhibits certain continuity properties in their spectrum 
useful for assessing their stability characteristics. To elaborate on this, let us write the characteristic function of the system as

$$
f\left(s, e^{-s \tau}\right) \triangleq \operatorname{det}\left[s I-A_{0}-A_{1} e^{-s \tau}\right]
$$

where $I$ is the identity matrix, $s$ is the Laplace variable, and the delay term $\tau$ appears in exponential in the Laplace sense. The zeros of (37), which are called the characteristic roots, determine the stability of the nominal system as follows. The nominal system is asymptotically stable for a given delay $\tau \geq 0$ and system matrices $A_{0}, A_{1}$, if and only if the characteristic roots all lie on the left-half complex plane $s \in \mathbb{C}$ [23].

Stability of (36) can be assessed with respect to $\tau$ by observing certain features of the system characteristic roots. The real part of these roots vary continuously with respect to the parameter $\tau$ and hence as $\tau$ is varied, the only way the system can switch from stable to unstable behavior, or vice versa, is that a root touches the imaginary axis at $s=\mp j \omega^{*}$ for some critical delays $\tau=\tau^{*}$ [26]. In general, as the critical delay is slightly increased, a pair of complex conjugate roots cross over the imaginary axis. Depending on the direction of crossing, the system will have two more, or two less, unstable roots.

Considering all the delays $\tau^{*}$ causing crossings over the imaginary axis, starting with $\tau=0$, one can decompose the delay axis into countably many intervals, where the upper/lower boundaries of each interval is determined by $\tau^{*}$, and neighboring intervals have two or more less unstable roots depending on the direction of crossing of the respective root $s=\mp j \omega^{*}$. Ultimately, the intervals for which the number of unstable roots is zero are labeled as stable, otherwise unstable. The principle behind this approach is known as the $\tau$-decomposition property $[27,28]$.

For stability assessment, it is crucial to detect all $\tau$ for which system characteristic roots touch the imaginary axis $s=\mp j \omega$. Notice however that this is not a trivial task mainly because the exponential terms in (37) make the system infinite dimensional. That is, there exist infinitely many roots of the system, and accurate and exhaustive detection of those touching the imaginary axis is a challenge. This very likely explains more than six decades of research on this particular problem, see a review in [29]. Without getting into details, here we mention that we will utilize the approach in [30] to compute the imaginary crossings and their corresponding delay values.

Comment 4 [Section 5.6.2, 32] (Fundamental Solution). Consider a system with single time-delay given by

$$
\dot{z}(t)=A_{0} z(t)+A_{1} z(t-\tau), \quad z(t)=\psi(t) \quad \text { for } \quad t \in[-\tau, 0]
$$

where $z(t) \in \mathbb{R}^{n}$ is the system state, $A_{0} \in \mathbb{R}^{n \times n}$ and $A_{1} \in \mathbb{R}^{n \times n}$ are constant matrices, and $\tau$ is a positive time-delay. Then, its solution satisfies

$$
z(t, \psi)=\Psi(t) \psi(0)+\int_{-\tau}^{0} \Psi(t-\tau-\theta) A_{1} \psi(\theta) \mathrm{d} \theta, \quad t \geq 0,
$$

where $\Psi(t) \in \mathbb{R}^{n \times n}$ is the fundamental solution satisfying

$$
\dot{\Psi}(t)=A_{0} \Psi(t)+A_{1} \Psi(t-\tau), \quad t \geq 0,
$$

and the initial condition $\Psi(0)=I$ and $\Psi(t)=0$ for $t<0$. Furthermore, assuming that the system given by (38) is asymptotically stable, then there exist an $\alpha>0$ such that

$$
\|\Psi(t)\|_{2} \leq K e^{-\alpha t}, \quad t \geq 0
$$


I for some $K>1$.

By applying Comment 4, one can bound the error dynamics given by (35) as

$$
\|\tilde{x}(t, \psi)\|_{2} \leq K\|\psi(0)\|_{2}+\frac{K}{\alpha}\left[\left\|A_{1}\right\|_{2} \bar{\psi}\left(e^{\alpha \tau}-1\right)+\left\|B_{1}\right\|_{2} \frac{\epsilon}{\sqrt{\lambda_{\min }(P)}}\right]
$$

where $\bar{\psi} \triangleq \sup _{-\tau \leq \theta \leq 0} \psi(\theta)$. Assuming that the initial condition of the system in (31) is equal to that of the ideal behavior of the system in (34) (i.e. $\bar{\psi}=0$ ), one can further simplify (42) as

$$
\|\tilde{x}(t, \psi)\|_{2} \leq \epsilon \mu, \quad \mu \triangleq \frac{K\left\|B_{1}\right\|_{2}}{\alpha \sqrt{\lambda_{\min }(P)}} .
$$

The upper bound on the error signal $\tilde{x}(t, \psi)$ obtained in (43) implies that the user-defined performance parameter $\epsilon$ can be utilized to control the deviation of the system from the ideal behavior in (34).

\section{Illustrative Numerical Example}

In this section, we demonstrate the efficacy of the presented architecture for an adaptive flight control application with a Neal-Smith pilot model. For this purpose, consider the linearized longitudinal flight dynamics of a generic hypersonic vehicle $[3,33]$ given by

$$
\dot{x}_{\mathrm{g}}(t)=A_{\mathrm{g}} x_{\mathrm{g}}(t)+B_{\mathrm{g}} u_{\mathrm{g}}(t), \quad x_{\mathrm{g}}(0)=x_{\mathrm{g}_{0}},
$$

where $x_{\mathrm{g}}(t)=[v(t), \alpha(t), q(t), \theta(t)]^{\mathrm{T}} \in \mathbb{R}^{4}$, with $v(t)$ being the velocity in feet per second, $\alpha(t)$ being the angle of attack in radians, $q(t)$ being the pitch rate in radians per second, and $\theta(t)$ being the pitch angle in radians. In addition, $u_{\mathrm{g}}=\left[u_{\mathrm{th}}(t), u_{\mathrm{e}}(t)\right]^{\mathrm{T}}$, where $u_{\mathrm{th}}(t)$ denotes the throttle equivalence ratio, and $u_{\mathrm{e}}(t)$ denotes the elevator deflection angle in degrees. The system matrices in (44) for steady level flight condition of Mach 6 and an altitude of 80,000 feet are given by

$$
A_{\mathrm{g}}=\left[\begin{array}{cccc}
-0.0037 & -0.7169 & 0 & -31.818 \\
0 & -0.2398 & 1 & 0 \\
0 & 4.5689 & -0.1189 & 0 \\
0 & 0 & 1 & 0
\end{array}\right], \quad B_{\mathrm{g}}=\left[\begin{array}{cc}
27.262 & 0.06525 \\
0 & -0.0001 \\
0 & -0.18561 \\
0 & 0
\end{array}\right]
$$

One can simplify this dynamics [3] and obtain the decoupled velocity dynamics given by

$$
\dot{v}(t)=a_{v} v(t)+b_{v} u_{\mathrm{th}},
$$

with $a_{v}=-0.0037$ and $b_{v}=27.262$, and the decoupled longitudinal dynamics given by

$$
\dot{x}^{*}(t)=\left[\begin{array}{cc}
A_{\mathrm{p}} & 0_{2 \times 2} \\
G_{\mathrm{p}} & F_{\mathrm{p}}
\end{array}\right] x^{*}(t)+\left[\begin{array}{c}
B_{\mathrm{p}} \\
0_{2 \times 1}
\end{array}\right]\left(\Lambda u_{\mathrm{e}}(t)+\delta_{\mathrm{p}}\left(x_{\mathrm{p}}(t)\right)\right),
$$

where $x^{*}(t)=\left[x_{\mathrm{p}}^{\mathrm{T}}(t), \phi_{\mathrm{p}}^{\mathrm{T}}(t)\right]^{\mathrm{T}} \in \mathbb{R}^{3}$ with $x_{\mathrm{p}}(t)=[\alpha(t), q(t)]^{\mathrm{T}} \in \mathbb{R}^{2}, \phi_{\mathrm{p}}(t)=\theta(t) \in \mathbb{R}$, and 


$$
A_{\mathrm{p}}=\left[\begin{array}{cc}
-0.2398 & 1 \\
4.5689 & -0.1189
\end{array}\right], \quad B_{\mathrm{p}}=\left[\begin{array}{c}
-0.0001 \\
-0.18561
\end{array}\right], G_{\mathrm{p}}=\left[\begin{array}{ll}
0 & 1
\end{array}\right], \quad F_{\mathrm{p}}=0
$$

In $(47), \delta_{\mathrm{p}}\left(x_{\mathrm{p}}(t)\right)$ represents an uncertainty of the form given in (4) with

$$
W_{\mathrm{p}}(t)=[20 \sin (0.05 t),-5,20]^{\mathrm{T}}, \quad \sigma_{\mathrm{p}}\left(x_{\mathrm{p}}(t)\right)=[\alpha(t), q(t), \alpha(t) q(t)]^{\mathrm{T}},
$$

and $\Lambda=0.25$ represents the uncertain control effectiveness.

Based on the model simplifications mentioned above, the control loop for the velocity dynamics can be designed independent from the longitudinal dynamics. For this purpose, we consider the velocity dynamics in (46) and we let $x_{\mathrm{vc}}(t) \in \mathbb{R}$ to be a velocity integrator state satisfying

$$
\dot{x}_{\mathrm{cv}}(t)=v(t)-c_{v}(t), \quad x_{\mathrm{vc}}(0)=0,
$$

such that the velocity can track the desired command $c_{v}(t)$. Considering this integral state, one can now define the augmented state vector as $x_{v}(t) \triangleq\left[v(t), x_{\mathrm{cv}}(t)\right]^{\mathrm{T}}$, and write

$$
\dot{x}_{v}(t)=\left[\begin{array}{cc}
a_{v} & 0 \\
1 & 0
\end{array}\right] x_{v}(t)+\left[\begin{array}{c}
b_{v} \\
0
\end{array}\right] u_{\mathrm{th}}(t)+\left[\begin{array}{c}
0 \\
-1
\end{array}\right] c_{v}(t), \quad x_{\mathrm{v}}(0)=0 .
$$

Linear quadratic regulator theory is used to design the nominal controller gain matrix for the velocity dynamics with the weighting matrices as $Q_{v}=\operatorname{diag}([10,1])$ to penalize $x_{v}(t)$ and $R_{v}=10$ to penalize $u_{\mathrm{th}}(t)$ resulting in $u_{\mathrm{th}}(t)=-K_{v} x_{v}(t)$ with

$$
K_{v}=\left[\begin{array}{ll}
1.0114 & 0.3162
\end{array}\right]
$$

In what follows, we design the controller for $u_{\mathrm{e}}(t)$ and provide the necessary details. Specifically, the control objective considered in this simulation is for the generic hypersonic vehicle to track the pitch angle $\theta_{\text {cmd }}(t)$ as commanded by the human. That is, the human is generating a pitch angle command (i.e., $\left.c_{0}(t)=\theta_{\text {cmd }}(t)\right)$, where the outer loop utilizes this command to generate the pitch rate command (i.e., $\left.c(t)=q_{\mathrm{cmd}}(t)\right)$. Then, using this pitch rate command, the inner loop generates the elevator command signal so that the system can track the desired pitch angle.

\section{A Inner Loop Control Design}

For command following at the inner loop using the pitch rate command $q_{\mathrm{cmd}}(t)$ generated by the outer loop, we consider the dynamic compensator in (5) and (6) with $A_{\mathrm{c}}=0, B_{\mathrm{c}}=1, C_{\mathrm{c}}=1, D_{\mathrm{c}}=0$, and $C_{\mathrm{p}}=\left[\begin{array}{ll}0 & 1\end{array}\right]$. We next use linear quadratic regulator theory to design the nominal controller gain matrix with the weighting matrices as $Q_{\mathrm{i}}=\operatorname{diag}([5,5,10])$ to penalize $x(t)$ and $R_{\mathrm{i}}=0.01$ to penalize $u_{\mathrm{e}}(t)$ resulting in $K_{\mathrm{p}}=$ [-36.3125, -34.4585], and $K_{\mathrm{c}}=-31.6228$ in (8). For the set-theoretic model reference adaptive control architecture at the inner loop, we use the generalized restricted potential function given by $\phi\left(\|e(t)\|_{P}\right)=$ $\|e(t)\|_{P}^{2} /\left(\epsilon-\|e(t)\|_{P}\right), e(t) \in \mathcal{D}_{\epsilon}$, having the partial derivative $\phi_{d}\left(\|e(t)\|_{P}\right)=\left(\epsilon-\frac{1}{2}\|e(t)\|_{P}\right) /\left(\epsilon-\|e(t)\|_{P}\right)^{2}$, $e(t) \in \mathcal{D}_{\epsilon}$, that satisfies all of the conditions given in Definition 2 [9]. Furthermore, we choose $\gamma=1$, set

the projection norm bound imposed on each element of the parameter estimate to $\hat{W}_{\max }=80$, use $R=I$ to calculate $P$ from (15) for the resulting $A_{\mathrm{r}}$ matrix, and set $\epsilon=0.1$ such that the set-theoretic model reference adaptive control guarantees $\|e(t)\|_{P}<0.1$. 


\section{B Outer Loop Control Design}

The outer loop utilizes the pitch command $\theta_{\text {cmd }}(t)$ from the human loop to generate the pitch rate command $q_{\mathrm{cmd}}(t)$ that is fed to the inner loop. For this purpose, we consider the feedback controller from $\theta_{\mathrm{cmd}}(t)$ to $q_{\mathrm{cmd}}(t)$ given by [3]

$$
G_{\theta q}=k_{\theta q} \frac{s+z_{\theta q}}{s+p_{\theta q}}
$$

For this numerical example, we set $k_{\theta q}=5, z_{\theta q}=1$, and $p_{\theta q}=4$.

\section{Human Loop Transfer Function}

To generate the pith command $\theta_{\text {cmd }}(t)$ at the human loop, we assume that the considered generic hypersonic vehicle is operated by a pilot whose Neal-Smith model is given by [18]

$$
G_{h \theta}=k_{p} \frac{T_{p} s+1}{T_{z} s+1} e^{-\tau s}
$$

where $k_{p}$ is the positive scalar pilot gain, $T_{p}$ and $T_{z}$ are positive scalar time constants, and $\tau$ is the pilot reaction time-delay. For the sake of this numerical example we set $k_{p}=0.5, T_{p}=1, T_{z}=5$ and $\tau=0.5$.

\section{Simulation Results}

We first construct the matrices $A_{0}$ and $A_{1}$ in Comment 3 to assess asymptotic stability with respect to delay $\tau$. To start with, it is easy to show that the delay-free system $(\tau=0)$ is asymptotically stable since $A_{0}+A_{1}$ is a Hurwitz matrix. Next, we investigate following from [30] whether or not a characteristic root $s=\mp j \omega$ can touch the imaginary axis for some delay $\tau>0$. Suppressing the details, we find out that there exists no $\tau>0$ that can cause a root on the imaginary axis. This implies that system stability will never be lost. That is, we have delay-independent stability. Consequently, the theoretical results for obtaining the upper bound on the error signal $\tilde{x}(t, \psi)$ in Section III can be validated in simulations for any delay.

Figures 2 and 3 show the pitch command following performance with the nominal controller in the absence of any system uncertainties, where it is clear from Figures 4 and 5 that once the uncertainties are introduced to the system, the nominal controller is not able to achieve the desired performance and the system becomes unstable. Next, we show the command following performance with the standard adaptive control architecture at the inner loop. As mentioned earlier, without the knowledge of the upper bound on the system uncertainties, an appropriate adaptation gain cannot be set a priori. Therefore, as one can see from Figures 6 to 8, while the standard model reference adaptive control architecture can stabilize the system, the desired level of system performance (i.e., $\|e(t)\|_{P}<0.1$ ) cannot be guaranteed. Specifically, since a low adaptation gain $\gamma=1$ is utilized, the standard model reference adaptive controller exhibits poor transient performance as the angle of attack $\alpha(t)$ reaches to over 20 degrees, pitch angle $\theta(t)$ reaches to over 30 degrees, and the pitch rate $q(t)$ reaches to over 40 degrees per second during the transient time. Furthermore, Figures 9 to 11 illustrate the effect of having different human reaction time-delays on the command following performance using this architecture. Once again, we note that, although increasing the adaptation gain can improve the transient performance in this setting, one cannot set a suitable adaptation gain at the pre-design stage without a complete knowledge of the upper bound on the system uncertainties. Alternatively, if a very large adaptation gain is used at all times, the adaptive control system can excite the high-frequency content of the system. 

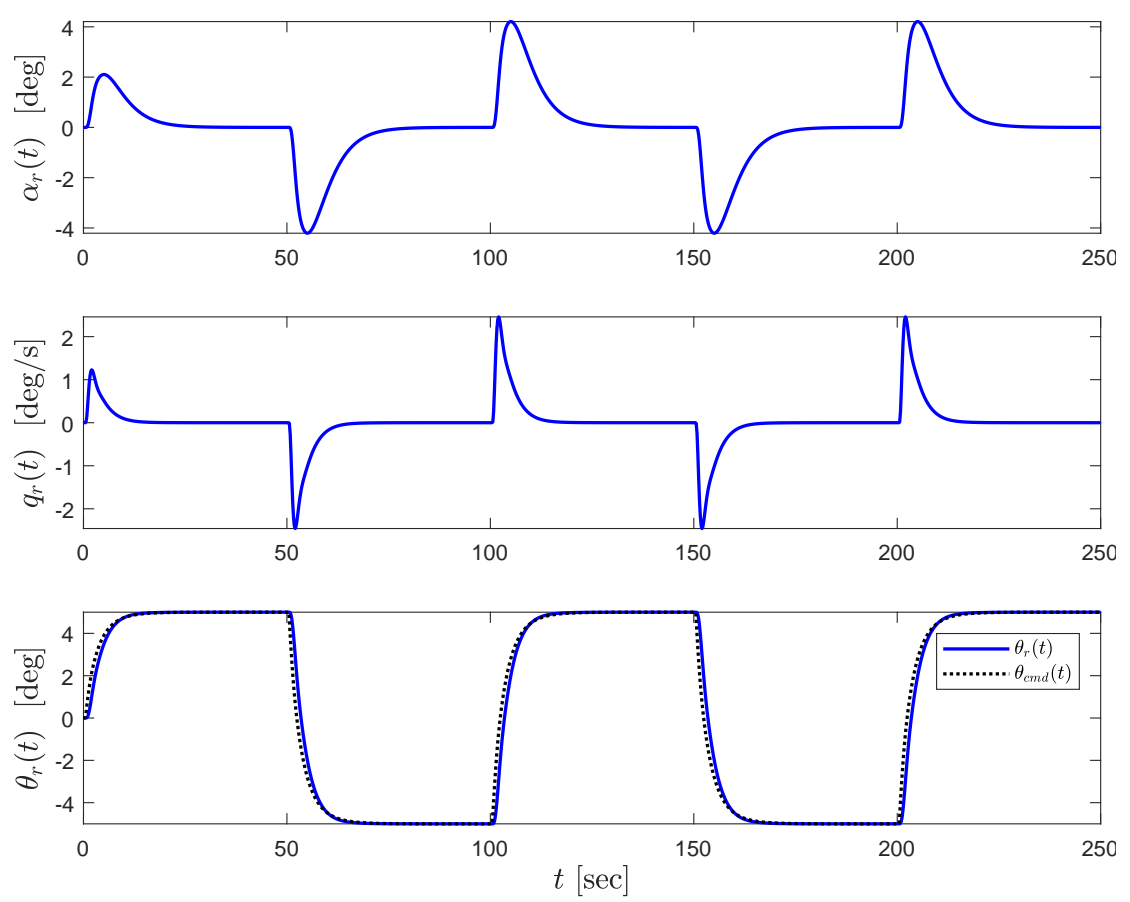

Figure 2. Command following performance with the nominal controller in the absence of the system uncertainty.
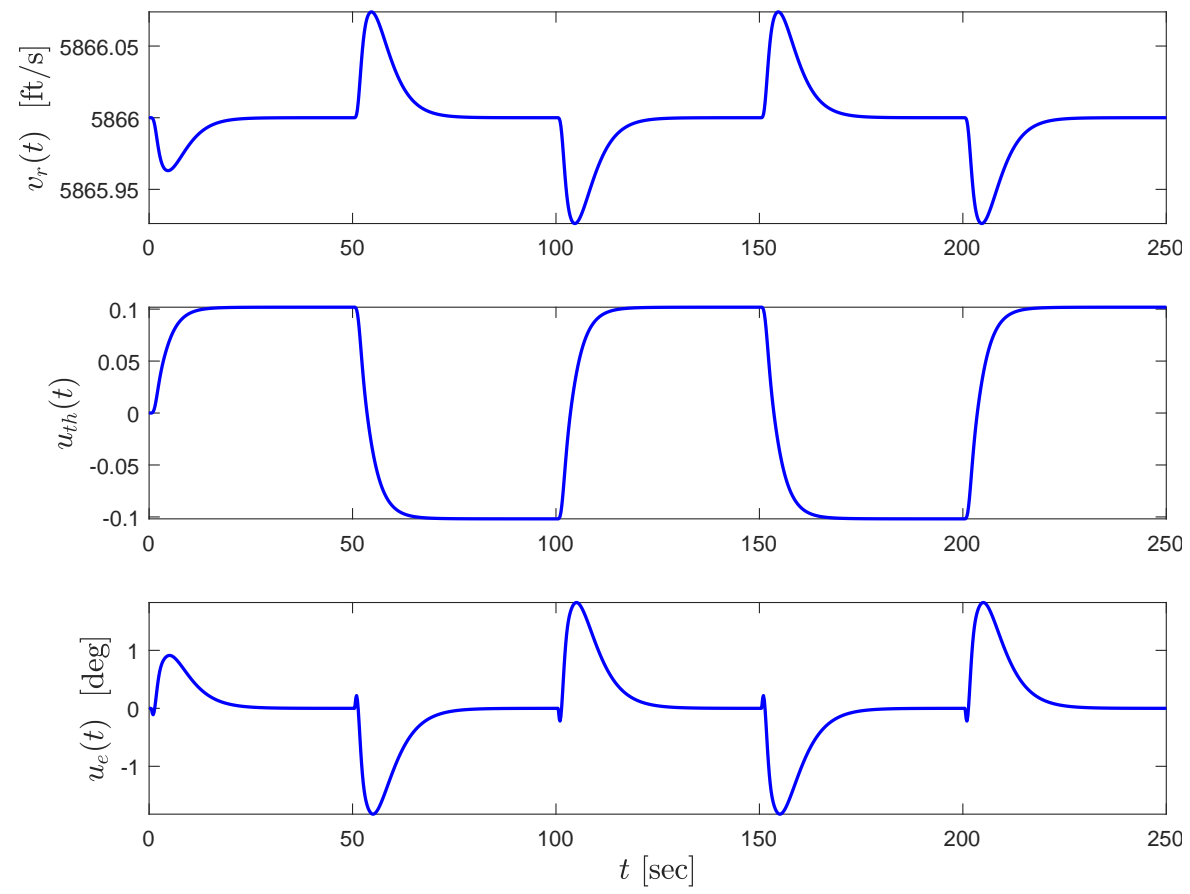

Figure 3. Velocity, altitude, and the control signals with the nominal controller in the absence of the system uncertainty. 

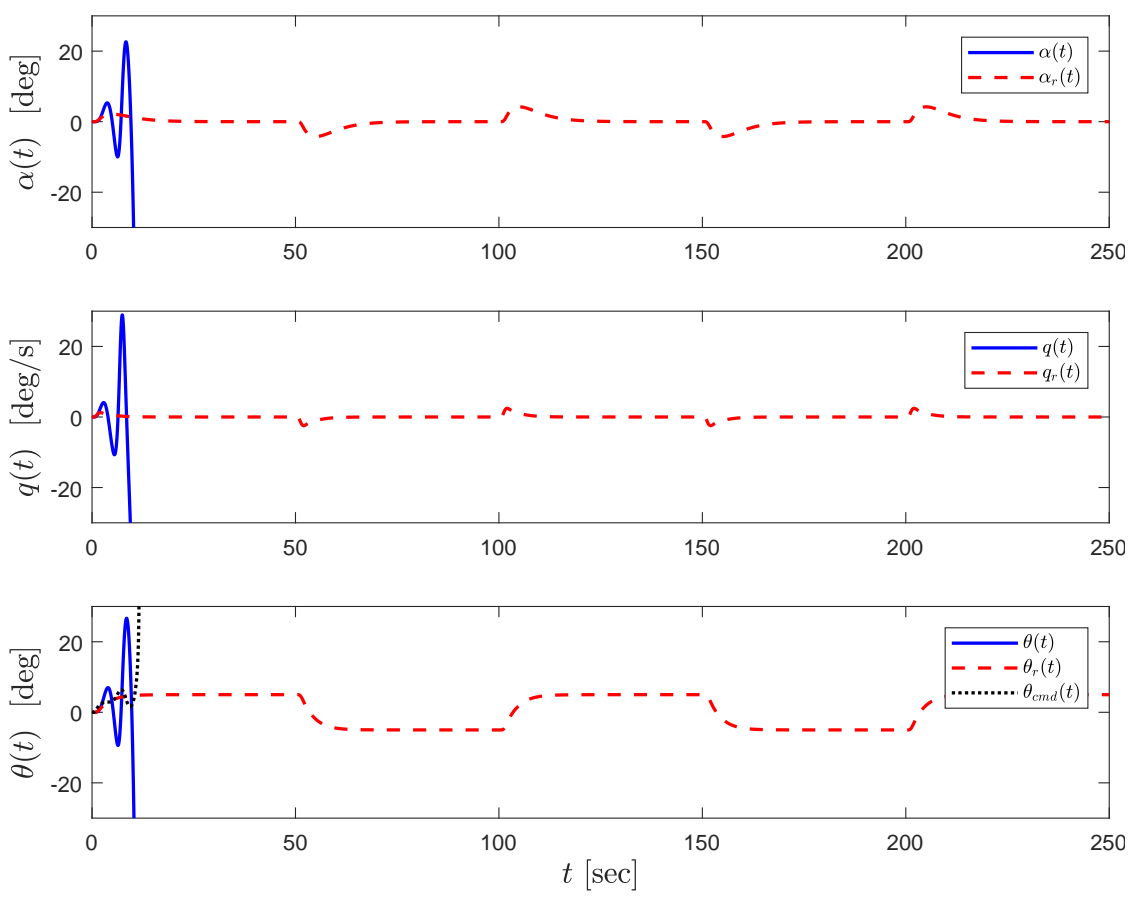

Figure 4. Command following performance with the nominal controller in the presence of the system uncertainty.
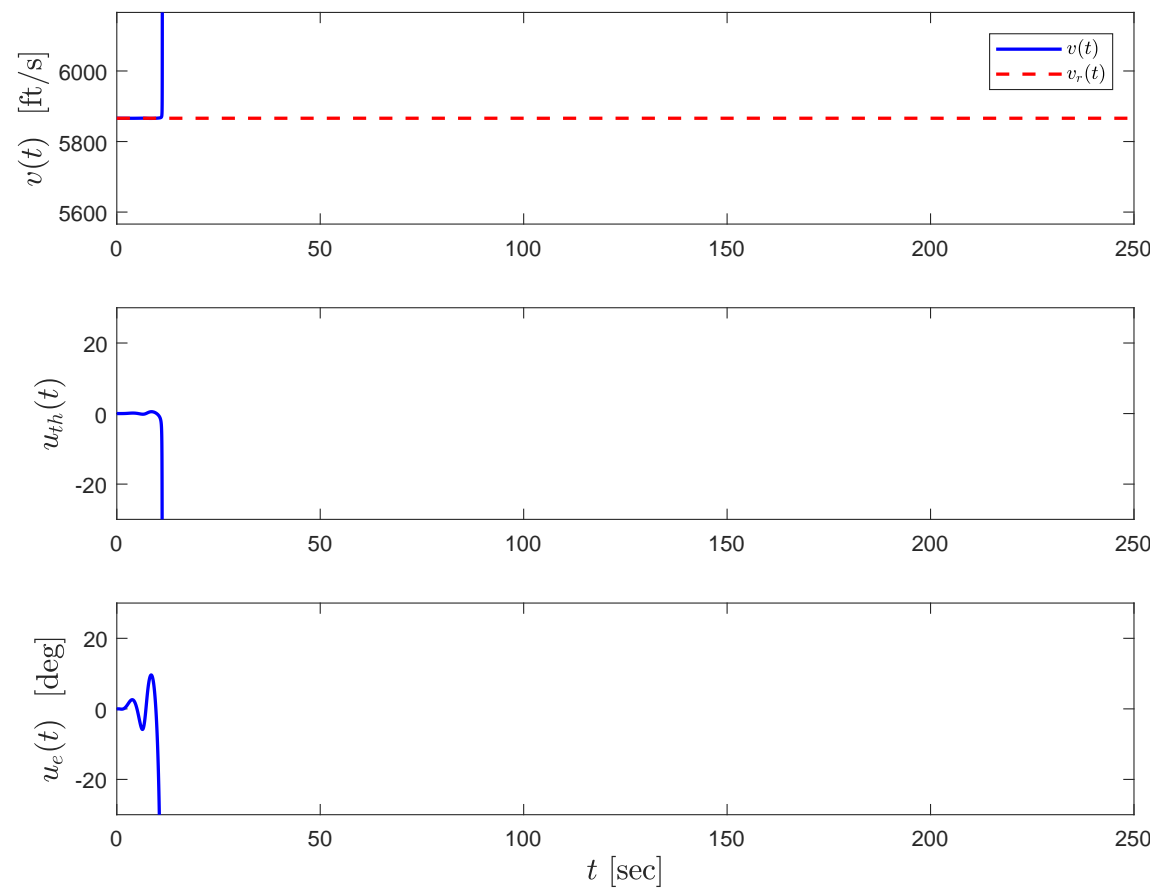

Figure 5. Velocity, altitude, and the control signals with the nominal controller in the presence of the system uncertainty. 

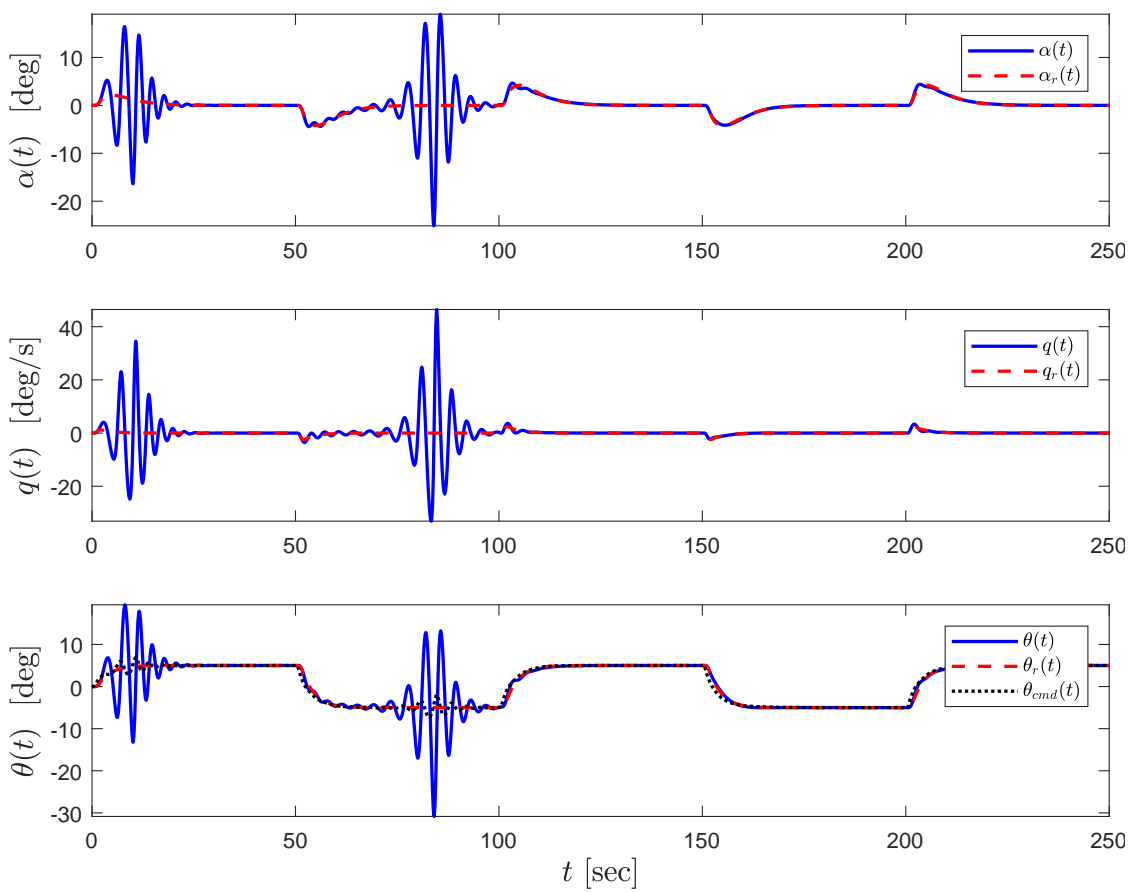

Figure 6. Command following performance with the standard model reference adaptive controller at the inner loop.
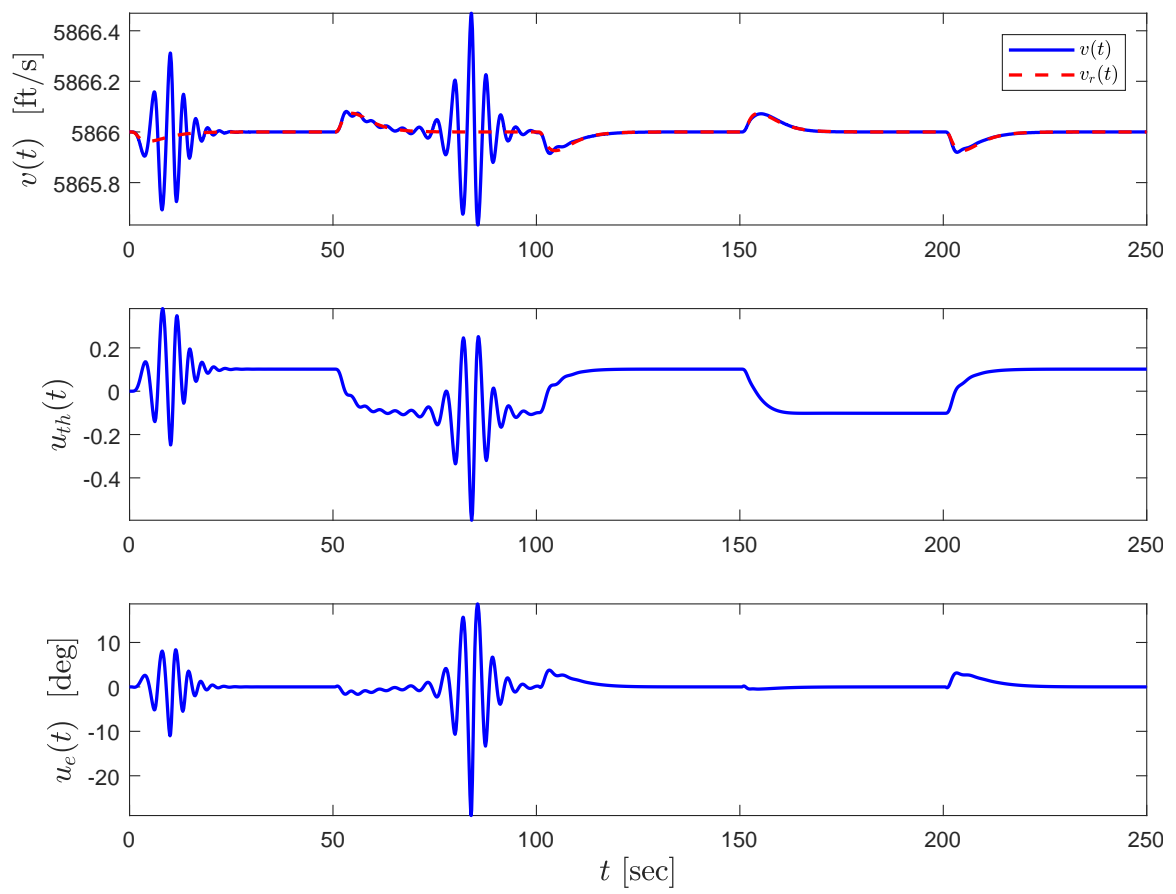

Figure 7. Velocity, altitude, and the control signals with the standard model reference adaptive controller at the inner loop. 

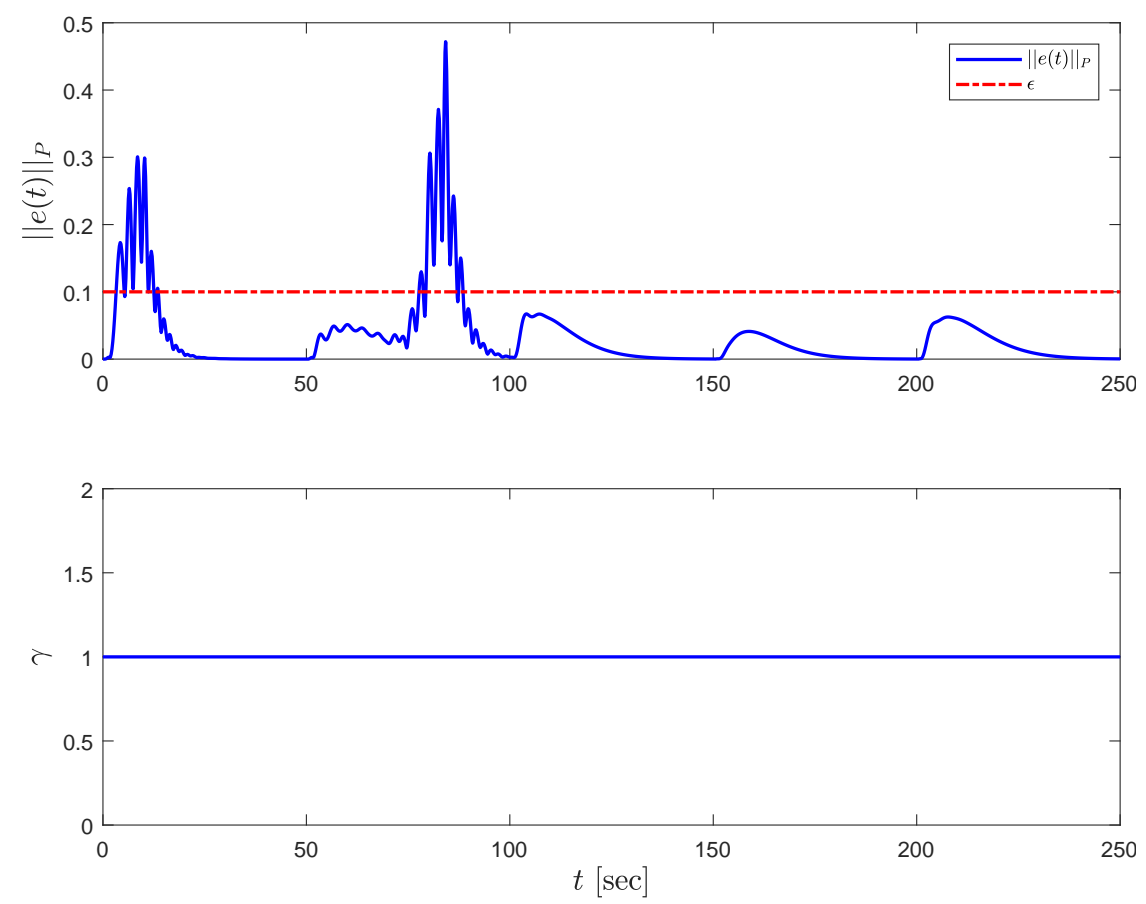

Figure 8. Norm of the system error trajectories with the standard model reference adaptive controller at the inner loop.
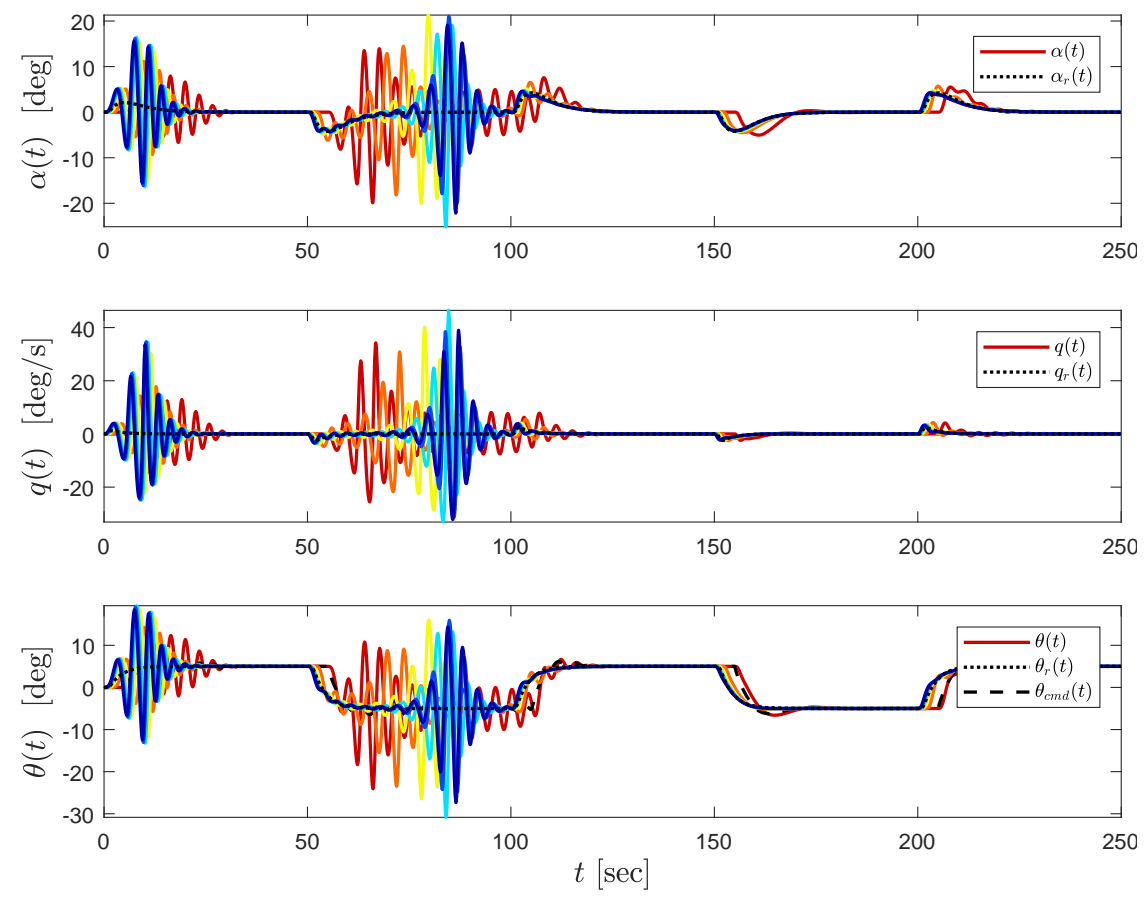

Figure 9. The effect of increase in the human reaction time-delay $\tau$ from 0 to 5 (blue to red) on the command following performance with the standard model reference adaptive controller at the inner loop. 

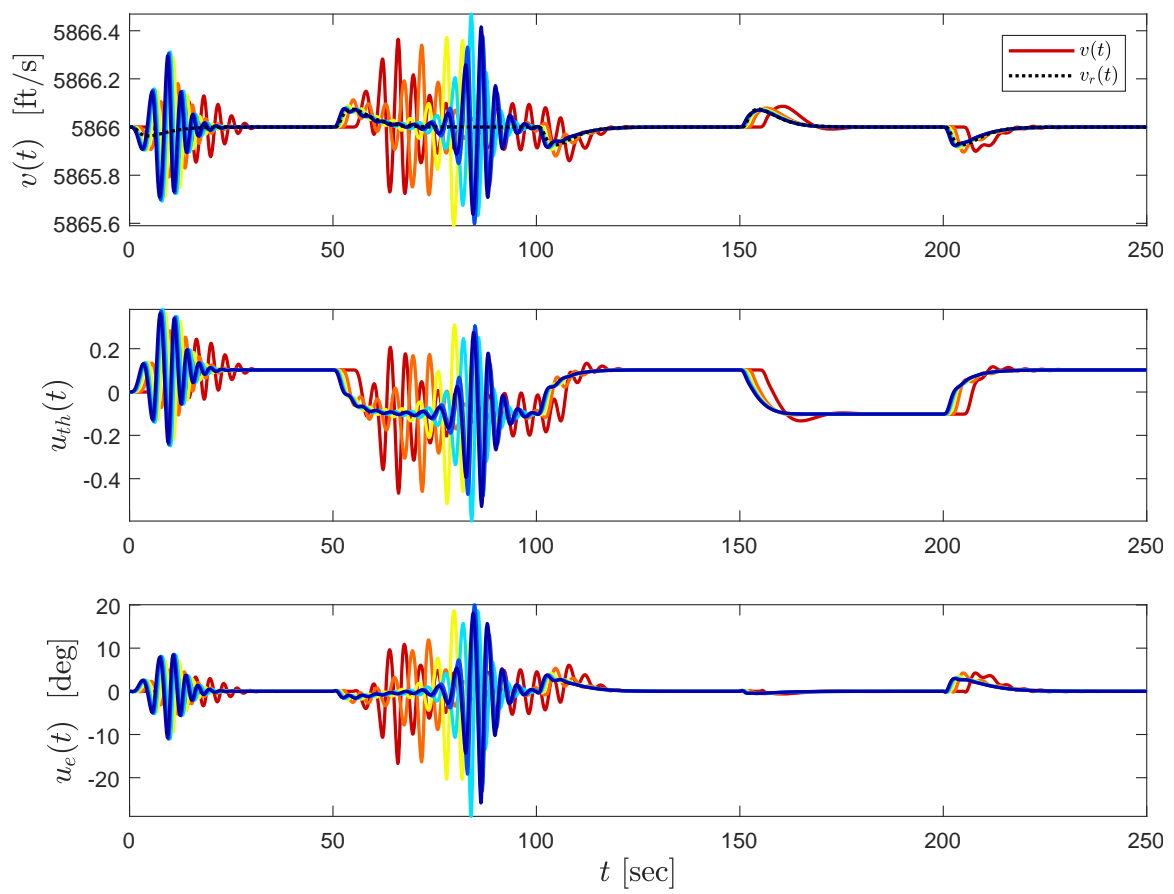

Figure 10. The effect of increase in the human reaction time-delay $\tau$ from 0 to 5 (blue to red) on velocity, altitude, and the control signals with the standard model reference adaptive controller at the inner loop.
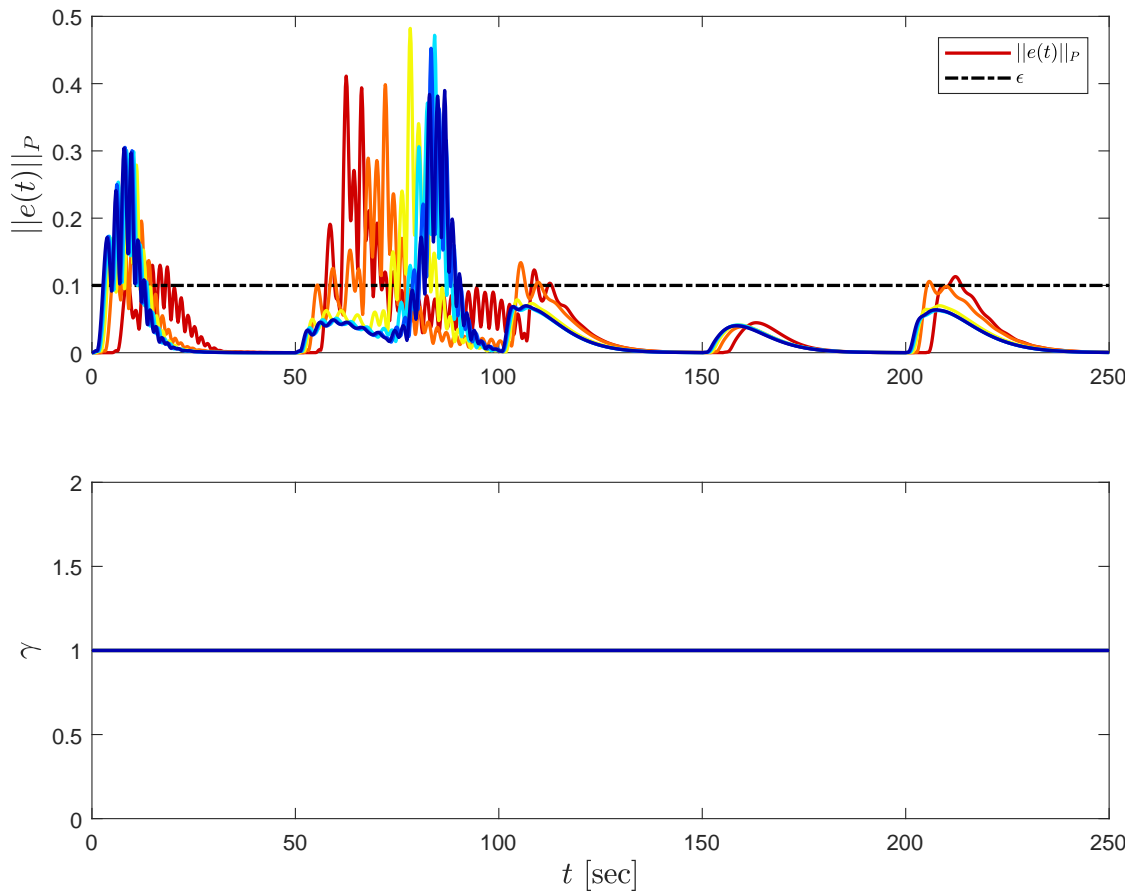

Figure 11. The effect of increase in the human reaction time-delay $\tau$ from 0 to 5 (blue to red) on the norm of the system error trajectories with the standard model reference adaptive controller at the inner loop. 
Next, we utilize the set-theoretic model reference adaptive control at the inner loop to enforce the desired system performance $\|e(t)\|_{P}<0.1$. Figures 12 to 14 present the command following performance with this controller. It can be seen from these figures that the transient performance is greatly improved compared to Figures 6 to 8. In particular, the set-theoretic model reference architecture at the inner loop is now enabling the overall control system to achieve the desired level of system performance (i.e., $\left.\|e(t)\|_{P}<0.1\right)$ through increasing the effective adaptation gain based on the norm of system error. Finally, Figures 15 to 17 present the effect of having different human reaction time-delays on the command following performance using the set-theoretic model reference adaptive control at the inner loop. It is evident from these figures that the proposed control architecture can guarantee a desired level of system performance even in the presence of large human reaction time-delays.

\section{Conclusion}

We studied human-in-the-loop physical systems with uncertainties due to failures and/or modeling inaccuracies, a set-theoretic model reference adaptive control law at the inner loop that augments a general nominal dynamic compensator structure, and a dynamic outer loop compensator to capture either sequential loop closure methods and/or high-level guidance algorithms. Specifically, to complement and extend our recent studies, we first provided a sufficient stability condition for the overall physical system; that is, asymptotic stability of the system given by (38) with $A_{0}$ and $A_{1}$ in (32). We then showed how to constrain the system error trajectories in order to minimally affect the performance of the overall human-in-the-loop physical system; that is, the upper bound given by (43) with $\epsilon$ denoting a user-defined constraint. Finally, we demonstrated the efficacy of our theoretical results through an illustrative numerical example.
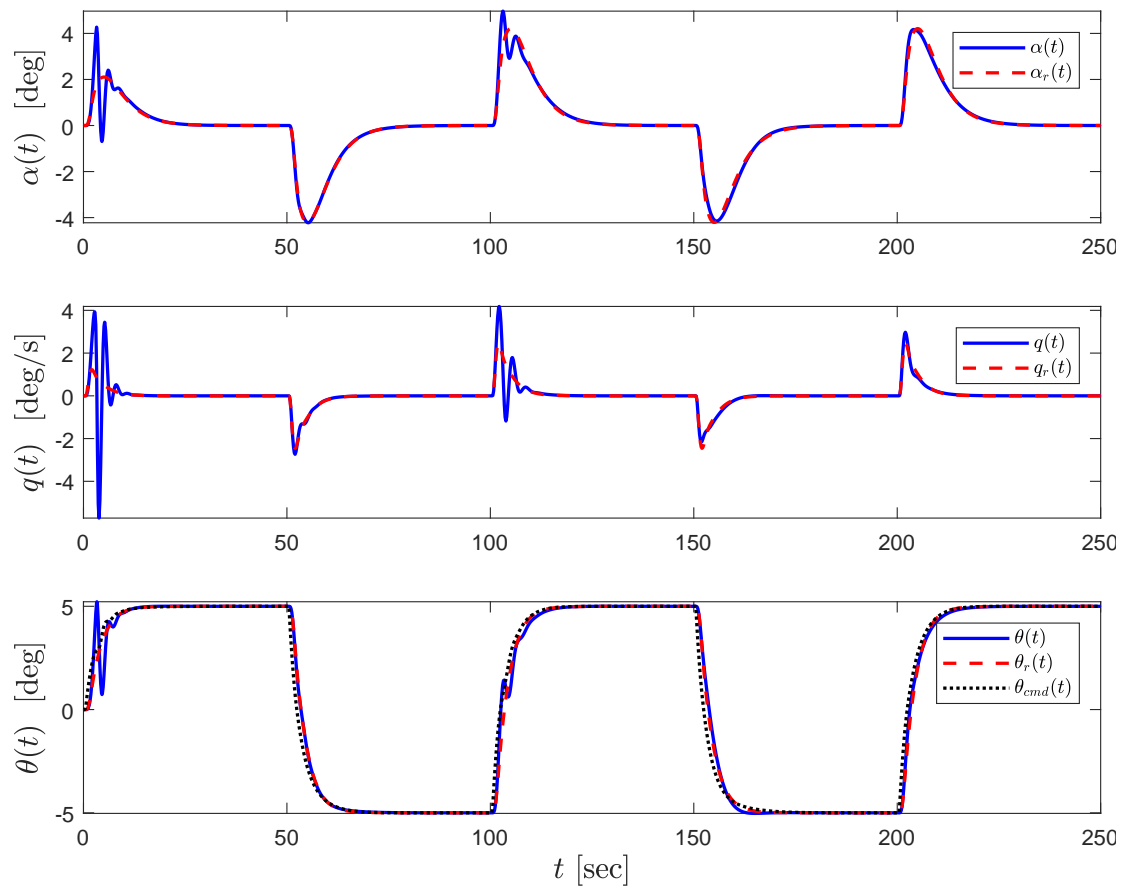

Figure 12. Command following performance with the proposed set-theoretic model reference adaptive controller at the inner loop. 

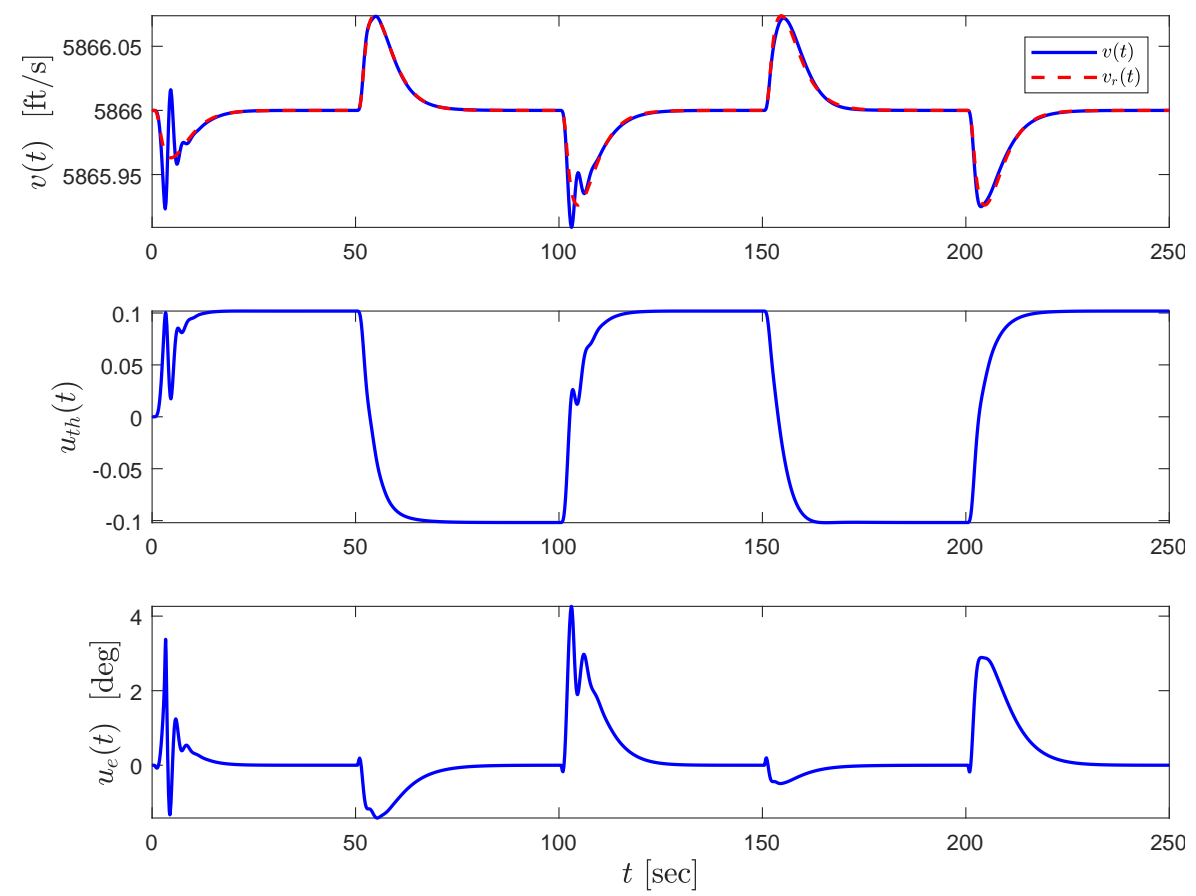

Figure 13. Velocity, altitude, and the control signals with the proposed set-theoretic model reference adaptive controller at the inner loop.
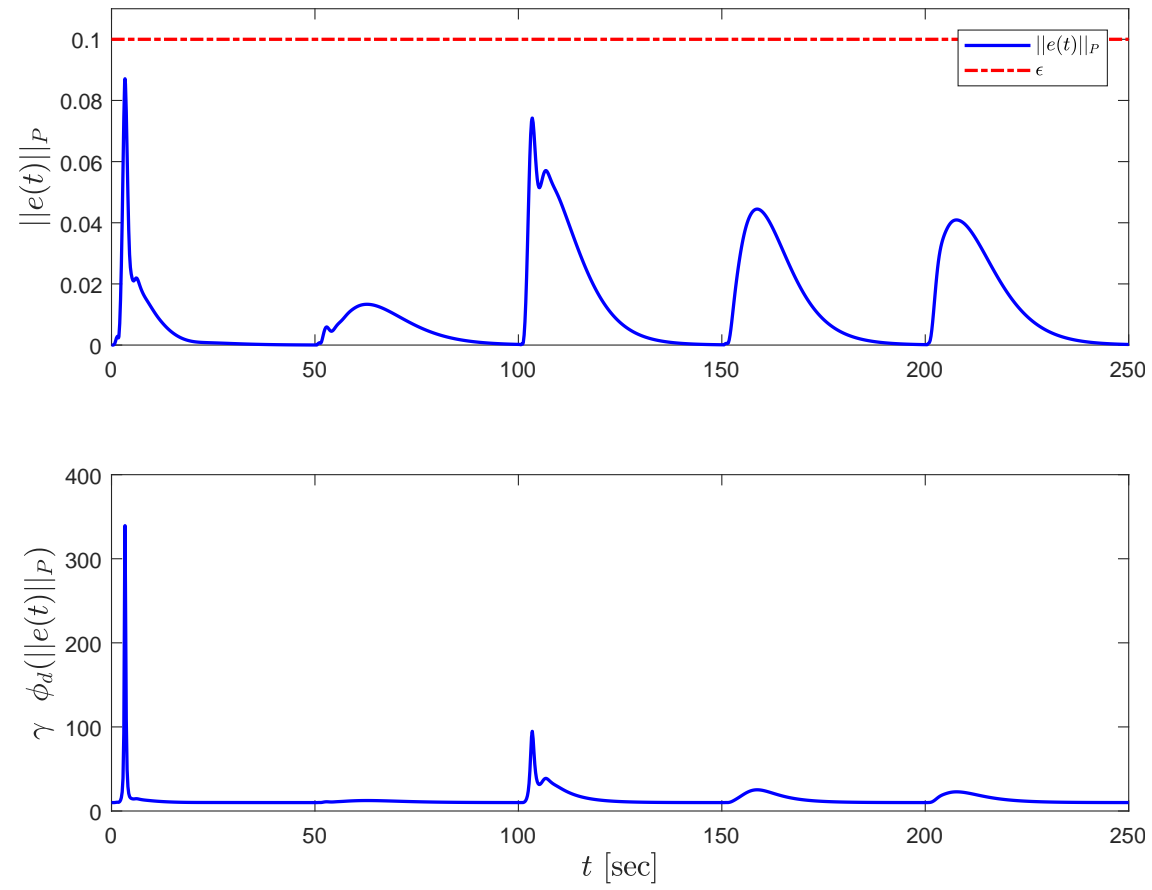

Figure 14. Norm of the system error trajectories and the evolution of the effective learning rate $\gamma \phi_{d}(\cdot)$ with the proposed set-theoretic model reference adaptive controller at the inner loop. 

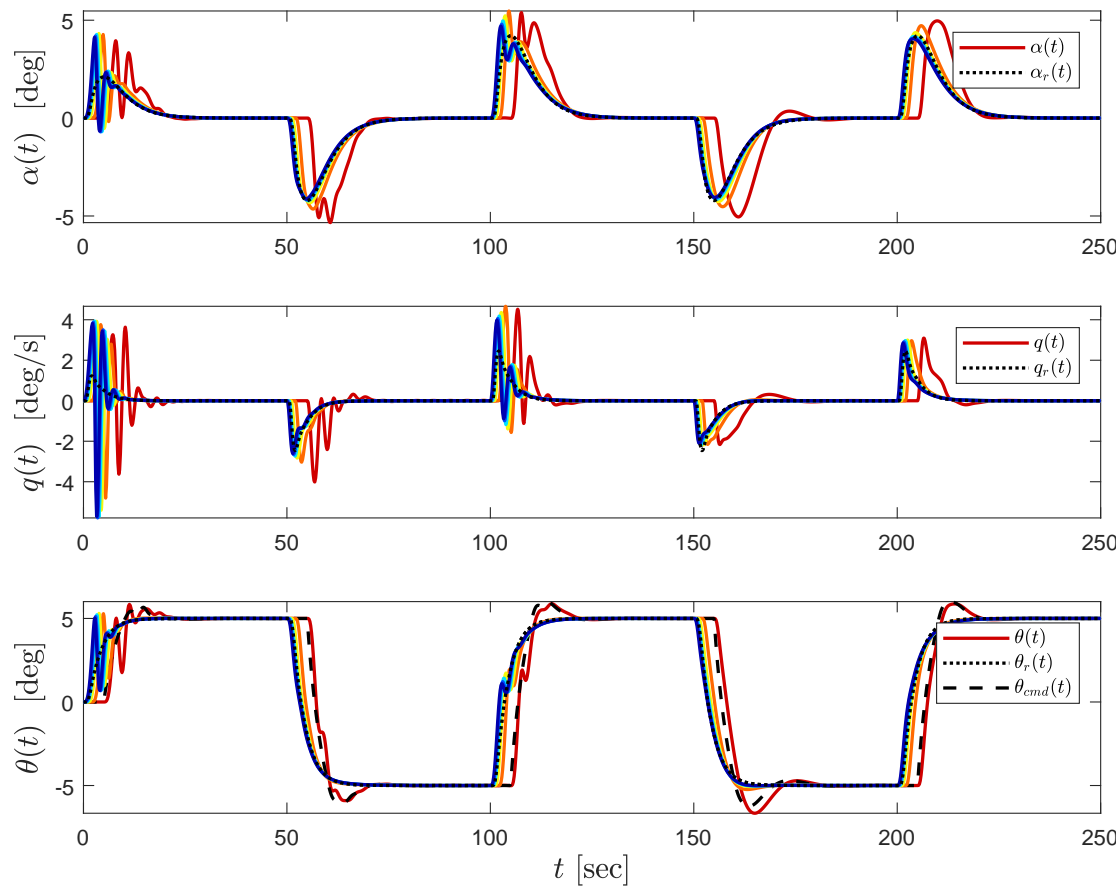

Figure 15. The effect of increase in the human reaction time-delay $\tau$ from 0 to 5 (blue to red) on the command following performance with the proposed set-theoretic model reference adaptive controller at the inner loop.
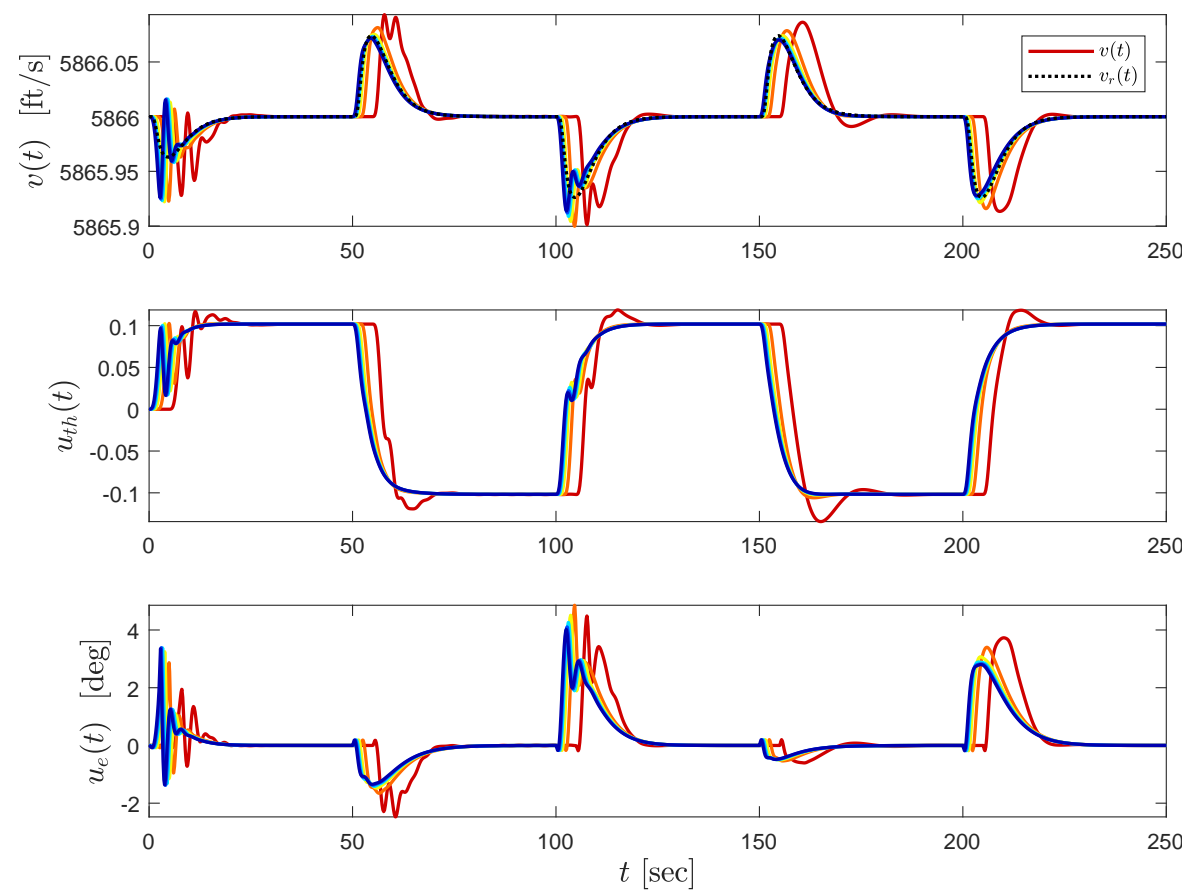

Figure 16. The effect of increase in the human reaction time-delay $\tau$ from 0 to 5 (blue to red) on velocity, altitude, and the control signals with the proposed set-theoretic model reference adaptive controller at the inner loop. 

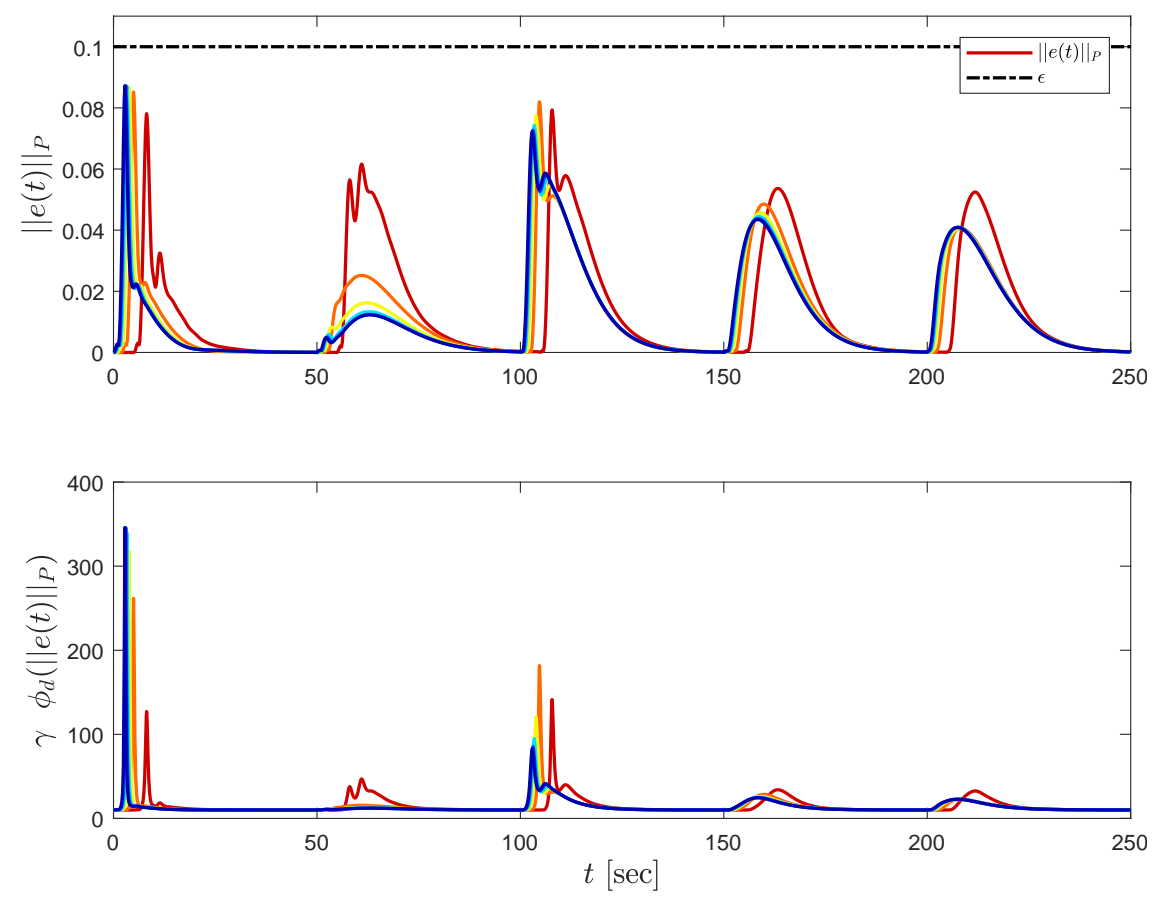

Figure 17. The effect of increase in the human reaction time-delay $\tau$ from 0 to 5 (blue to red) on the norm of the system error trajectories and the evolution of the effective learning rate $\gamma \phi_{d}(\cdot)$ with the proposed set-theoretic model reference adaptive controller at the inner loop.

\section{Acknowledgments}

Our appreciations to Prof. Keqin Gu (Department of Mechanical and Industrial Engineering, Southern Illinois University Edwardsville) for his time to provide feedback on our developments related to Comment 4 .

\section{References}

${ }^{1}$ T. Yucelen, Y. Yildiz, R. Sipahi, E. Yousefi, and N. Nguyen, "Stability limit of human-in-the-loop model reference adaptive control architectures," International Journal of Control, pp. 1-18, 2017.

${ }^{2}$ D. P. Wiese, "Systematic adaptive control design using sequential loop closure," Ph.D. dissertation, Massachusetts Institute of Technology, 2016.

${ }^{3}$ D. P. Wiese, A. M. Annaswamy, J. A. Muse, M. A. Bolender, and E. Lavretsky, "Sequential loop closure based adaptive autopilot design for a hypersonic vehicle," in AIAA Guidance, Navigation, and Control Conference, 2016, p. 1379.

${ }^{4}$ D. T. McRuer, "Mathematical models of human pilot behavior," 1974.

${ }^{5}$ M. Green, “'How Long Does It Take to Stop?' methodological analysis of driver perception-brake times," Transportation Human Factors, vol. 2, pp. 195-216, 2000.

${ }^{6}$ D. Helbing, "Traffic and related self-driven many-particle systems," Reviews of Modern Physics, vol. 73, pp. 1067-1141, 2001.

${ }^{7}$ M. Treiber, A. Kesting, and D. Helbing, "Delays, inaccuracies and anticipation in microscopic traffic models," Physica $A$, vol. 360, no. 1, pp. 71-88, 2006.

${ }^{8}$ G. Stépán, Delay effects in brain dynamics. Philosophical Transactions of The Royal Society A - Mathematical Physical \& Engineering Sciences, 2009, vol. 367, no. 1891.

${ }^{9}$ E. Arabi, B. C. Gruenwald, T. Yucelen, and N. T. Nguyen, "A set-theoretic model reference adaptive control architecture for disturbance rejection and uncertainty suppression with strict performance guarantees," International Journal of Control, 2018.

${ }^{10} \mathrm{E}$. Arabi and T. Yucelen, "Set-theoretic model reference adaptive control with time-varying performance bounds," International Journal of Control, (accepted-available online). 
11 __ "Generalization to set-theoretic model reference adaptive control architecture for enforcing user-defined time-varying performance bounds," American Control Conference, 2017.

${ }^{12}$ E. Arabi, T. Yucelen, B. C. Gruenwald, M. L. Fravolini, S. Balakrishnan, and N. T. Nguyen, "A neuroadaptive architecture for model reference control of uncertain dynamical systems with performance guarantees," Systems $E$ Control Letters, (under review).

${ }^{13}$ E. Arabi, B. C. Gruenwald, T. Yucelen, M. L. Fravolini, and N. T. Nguyen, "Model reference neuroadaptive control revisited: How to keep the system trajectories on a given compact set," in AIAA Guidance, Navigation, and Control Conference, 2017.

${ }^{14}$ E. Arabi, B. C. Gruenwald, T. Yucelen, and J. E. Steck, "Guaranteed model reference adaptive control performance in the presence of actuator failures," in AIAA Guidance, Navigation, and Control Conference, 2017.

${ }^{15} \mathrm{E}$. Arabi and T. Yucelen, "On set-theoretic model reference adaptive control of uncertain dynamical systems subject to actuator dynamics," in AIAA Guidance, Navigation, and Control Conference, 2018.

${ }^{16} \mathrm{E}$. Lavretsky and K. Wise, Robust and adaptive control with aerospace applications. Springer Science \& Business Media, 2012.

${ }^{17}$ J.-B. Pomet and L. Praly, "Adaptive nonlinear regulation: Estimation from the lyapunov equation," IEEE Transactions on Automatic Control, vol. 37, no. 6, pp. 729-740, 1992.

${ }^{18}$ D. Schmidt and B. Bacon, "An optimal control approach to pilot/vehicle analysis and the neal-smith criteria," Journal of Guidance Control Dynamics, vol. 6, pp. 339-347, 1983.

${ }^{19}$ A. J. Thurling, "Improving uav handling qualities using time delay compensation," Air Force Inst of Tech WrightPatterson AFB, Tech. Rep., 2000.

${ }^{20}$ S. Ryu and D. Andrisani, "Longitudinal flying qualities prediction for nonlinear aircraft," Journal of Guidance Control and Dynamics, vol. 26, no. 3, pp. 474-482, 2003.

${ }^{21} \mathrm{~J}$. B. Witte, "An investigation relating longitudinal pilot-induced oscillation tendency rating to describing function predictions for rate-limited actuators," Air Force Inst of Tech Wright-Patterson AFB, Tech. Rep., 2004.

${ }^{22}$ C. J. Miller, "Nonlinear dynamic inversion baseline control law: architecture and performance predictions," in AIAA Guidance, Navigation, and Control Conference, 2011, p. 6467.

${ }^{23}$ R. E. Bellman and K. L. Cooke, Differential-Difference Equations. Academic Press, 1963.

${ }^{24}$ G. Stépán and T. Insperger, Semi-Discretization for Time-Delay Systems: Stability and Engineering Applications. Springer, 2011.

${ }^{25}$ J. K. Hale and S. M. V. Lunel, Introduction to Functional Differential Equations. Springer-Verlag, 1993.

${ }^{26} \mathrm{R}$. Datko, "A procedure for determination of the exponential stability of certain differential-difference equations," Quarterly Applied Mathematics, vol. 36, pp. 279-292, 1978.

${ }^{27}$ J. Neimark, "D-subdivisions and spaces of quasi-polynomials," Prikl. Mat Meh., vol. 13, pp. 349-380, 1949.

${ }^{28}$ L. E. El'sgol'ts and S. B. Norkin, Introduction to the Theory and Applications of Differential Equations with Deviating Arguments. Academic Press, 1973.

${ }^{29}$ R. Sipahi, S.-I. Niculescu, C. T. Abdallah, W. Michiels, and K. Gu, "Stability and stabilization of systems with time delay, limitations and opportunities," IEEE Control Systems Magazine, pp. 38-65, 2011.

${ }^{30} \mathrm{~J}$. Louisell, "A matrix method for determining the imaginary axis eigenvalues of a delay system," IEEE Transactions on Automatic Control, vol. 46, pp. 2008-2012, 2001.

${ }^{31}$ D. Breda, S. Maset, and R. Vermiglio, Stability of Linear Delay Differential Equations: A Numerical Approach with MATLAB. SpringerBriefs in Control Automation and Robotics, 2015.

${ }^{32}$ K. Gu, J. Chen, and V. L. Kharitonov, Stability of time-delay systems. Springer Science \& Business Media, 2003.

${ }^{33}$ R. F. Stengel, Flight dynamics. Princeton University Press, 2004.

\section{Appendices}

\section{A Notation}

The notation used throughout this paper is consistent with our prior work [1]. Specifically, $\mathbb{R}$ denotes the set of real numbers, $\mathbb{C}$ denotes the set of complex numbers, $\mathbb{R}^{n}$ denotes the set of $n \times 1$ real column vectors, $\mathbb{R}^{n \times m}$ denotes the set of $n \times m$ real matrices, $\mathbb{R}_{+}$denotes the set of positive real numbers, $\mathbb{R}_{+}^{n \times n}$ denotes 
the set of $n \times n$ positive definite matrices, $\mathbb{D}^{n \times n}$ denotes the set of $n \times n$ real matrices with diagonal scalar entries, $0_{n \times n}$ denotes the $n \times n$ zero matrix, and "ڤ” denotes equality by definition. In addition, we write $(\cdot)^{\mathrm{T}}$ for the transpose, $(\cdot)^{-1}$ for the inverse, $\operatorname{tr}(\cdot)$ for the trace, $\|\cdot\|_{2}$ for the Euclidean norm, $\|\cdot\|_{\mathrm{F}}$ for the Frobenius norm, and $\|A\|_{2} \triangleq \sqrt{\lambda_{\max }\left(A^{\mathrm{T}} A\right)}$ for the induced 2-norm of the matrix $A \in \mathbb{R}^{n \times m}$.

\section{B Necessary Definitions}

The following definitions are used in the main results of this paper.

Definition $1[16,17]$ (Projection Operator). Let $\Omega=\left\{\theta \in \mathbb{R}^{n}:\left(\theta_{i}^{\min } \leq \theta_{i} \leq \theta_{i}^{\max }\right)_{i=1,2, \cdots, n}\right\}$ be a convex hypercube in $\mathbb{R}^{n}$, where $\left(\theta_{i}^{\min }, \theta_{i}^{\max }\right)$ represent the minimum and maximum bounds for the $i^{\text {th }}$ component of the $n$-dimensional parameter vector $\theta$. In addition, for a sufficiently small positive constant $\nu$, a second hypercube is defined by $\Omega_{\nu}=\left\{\theta \in \mathbb{R}^{n}:\left(\theta_{i}^{\min }+\nu \leq \theta_{i} \leq \theta_{i}^{\max }-\nu\right)_{i=1,2, \cdots, n}\right\}$, where $\Omega_{\nu} \subset \Omega$. The projection operator Proj: $\mathbb{R}^{n} \times \mathbb{R}^{n} \rightarrow \mathbb{R}^{n}$ is then defined component-wise by $\operatorname{Proj}(\theta, y) \triangleq\left(\frac{\theta_{i}^{\max }-\theta_{i}}{\nu}\right) y_{i}$, if $\theta_{i}>\theta_{i}^{\max }-\nu$ and $y_{i}>0, \operatorname{Proj}(\theta, y) \triangleq\left(\frac{\theta_{i}-\theta_{i}^{\min }}{\nu}\right) y_{i}$, if $\theta_{i}<\theta_{i}^{\min }+\nu$ and $y_{i}<0$, and $\operatorname{Proj}(\theta, y) \triangleq y_{i}$, otherwise, where $y \in \mathbb{R}^{n}$ [16]. Based on this definition and $\theta^{*} \in \Omega_{\nu}$, note that

$$
\left(\theta-\theta^{*}\right)^{\mathrm{T}}(\operatorname{Proj}(\theta, y)-y) \leq 0
$$

holds for $\theta \in \Omega$ and $y \in \mathbb{R}^{n}[16,17]$. This definition can be further generalized to matrices as $\operatorname{Proj}_{\mathrm{m}}(\Theta, Y)=$ $\left(\operatorname{Proj}\left(\operatorname{col}_{1}(\Theta), \operatorname{col}_{1}(Y)\right), \ldots, \operatorname{Proj}\left(\operatorname{col}_{m}(\Theta), \operatorname{col}_{m}(Y)\right)\right)$, where $\Theta \in \mathbb{R}^{n \times m}, Y \in \mathbb{R}^{n \times m}$ and $\operatorname{col}_{i}(\cdot)$ denotes $i$ th column operator. In this case, for a given matrix $\Theta^{*}$ it follows from (B.1) that $\operatorname{tr}\left[\left(\Theta-\Theta^{*}\right)^{\mathrm{T}}\left(\operatorname{Proj}_{\mathrm{m}}(\Theta, Y)-\right.\right.$ $Y)]=\sum_{i=1}^{m}\left[\operatorname{col}_{i}\left(\Theta-\Theta^{*}\right)^{\mathrm{T}}\left(\operatorname{Proj}\left(\operatorname{col}_{i}(\Theta), \operatorname{col}_{i}(Y)\right)-\operatorname{col}_{i}(Y)\right)\right] \leq 0$.

Definition 2 [9] (Generalized Restricted Potential Function). Let $\|z\|_{H}=\sqrt{z^{\mathrm{T}} H z}$ be a weighted Euclidean norm, where $z \in \mathbb{R}^{p}$ is a real column vector and $H \in \mathbb{R}_{+}^{p \times p}$. We define $\phi\left(\|z\|_{\mathrm{H}}\right), \phi: \mathbb{R} \rightarrow \mathbb{R}$, to be a generalized restricted potential function (generalized barrier Lyapunov function) on the set

$$
\mathcal{D}_{\epsilon} \triangleq\left\{z:\|z\|_{\mathrm{H}} \in[0, \epsilon)\right\}
$$

with $\epsilon \in \mathbb{R}_{+}$being a-priori, user-defined constant, if the following statements hold [9]: i) If $\|z\|_{\mathrm{H}}=0$, then $\phi\left(\|z\|_{\mathrm{H}}\right)=0$. ii) If $z \in \mathcal{D}_{\epsilon}$ and $\|z\|_{\mathrm{H}} \neq 0$, then $\phi\left(\|z\|_{\mathrm{H}}\right)>0$. iii) If $\|z\|_{\mathrm{H}} \rightarrow \epsilon$, then $\phi\left(\|z\|_{\mathrm{H}}\right) \rightarrow \infty$. iv) $\phi\left(\|z\|_{\mathrm{H}}\right)$ is continuously differentiable on $\mathcal{D}_{\epsilon}$. v) If $z \in \mathcal{D}_{\epsilon}$, then $\phi_{d}\left(\|z\|_{\mathrm{H}}\right)>0$, where $\phi_{d}\left(\|z\|_{\mathrm{H}}\right) \triangleq$ $\mathrm{d} \phi\left(\|z\|_{\mathrm{H}}\right) / \mathrm{d}\|z\|_{\mathrm{H}}^{2}$. vi) If $z \in \mathcal{D}_{\epsilon}$, then $2 \phi_{d}\left(\|z\|_{\mathrm{H}}\right)\|z\|_{\mathrm{H}}^{2}-\phi\left(\|z\|_{\mathrm{H}}\right)>0$. 